\title{
Humidity Sensing Enhancement and Structural Evolution of Tungsten Doped Zinc Oxide Nano- Sensors Fabricated Through Co-Precipitation Synthesis
}

Vernica Verma ( $\nabla$ vernicaverma20@gmail.com )

University of Lucknow https://orcid.org/0000-0003-4589-5269

Narendra Kumar Pandey

University of Lucknow Faculty of Science

Priya Gupta

Integral University

Kuldeep Singh

University of Lucknow

Peramjeet Singh

University of Lucknow

\section{Original Research}

Keywords: Humidity sensors, Tungsten doped ZnO, Nano-scaled, Sensitivity, Hysteresis.

Posted Date: February 5th, 2021

DOI: https://doi.org/10.21203/rs.3.rs-162049/v1

License: (c) (1) This work is licensed under a Creative Commons Attribution 4.0 International License.

Read Full License 


\title{
Humidity Sensing Enhancement and Structural Evolution of Tungsten Doped Nano- Sensors Fabricated Through Co-Precipitation Synthesis
}

\author{
Vernica Verma ${ }^{1 *}$, Narendra Kumar Pandey ${ }^{1}$, Priya Gupta ${ }^{2}$, Kuldeep Singh $^{1}$, Peramjeet Singh ${ }^{1}$ \\ ${ }^{1}$ Department of Physics, University of Lucknow, Lucknow, U.P.-226007, India
}

${ }^{2}$ Electronics and Communication Engineering Department, Integral University, Lucknow,

U.P. -226026, India.

*Corresponding author:

Email address: vernicaverma20@gmail.com

\begin{abstract}
Nano-sensors samples of pure Zinc oxide and tungsten doped $\mathrm{ZnO}$ are employed for the study of their humidity sensing performance, morphological and micro-structural details. The nano-powder for sensors of $\mathrm{ZnO}_{1-\mathrm{x}} \mathrm{W}_{\mathrm{x}}(\mathrm{x}=0$ to $1.75 \mathrm{~mol} \%)$ are prepared in molar ratios of $0.50 \mathrm{~mol} \%, 1.25 \mathrm{~mol} \%$, and $1.75 \mathrm{~mol} \%$ synthesized through co-precipitation technique. The effect of tungsten incorporation in $0.50 \mathrm{~mol} \%, 1.25 \mathrm{~mol} \%$ and $1.75 \mathrm{~mol} \%$ on the structural and morphological properties of nanostructures has been investigated via XRD and SEM, respectively. The $\mathrm{XRD}$ of the synthesized $\mathrm{ZnO}$ nanomaterial possess hexagonal wurtzite structure for all the samples. The obtained diffractogram depicts high and broad peaks implying good crystallinity and smaller crystallite size. The molarity of the dopants yields sufficient reduction in crystallite size from $44.46 \mathrm{~nm}$ to $37.38 \mathrm{~nm}$. The crystallite size of the samples
\end{abstract}


calculated using W-H analysis yields $44.28 \mathrm{~nm}$ to $39.37 \mathrm{~nm}$. The strain $(\epsilon)$ and stress $(\sigma)$ produced in the crystal due to dopants is analyzed via USDM, UDEDM and SSP thereby deducing energy density ' $u$ '. The pelletized nano-sensors of diameter $10 \mathrm{~mm}$ and thickness $2 \mathrm{~mm}$ show excellent response towards humidity sensing within the range of $10 \%$ to $90 \%$ of relative humidity. An appreciable sensitivity of $144 \mathrm{x}$ and hysteresis of 8.24 is exhibited $1.75 \mathrm{~mol} \% \mathrm{~W}$ $\mathrm{ZnO}$ humidity sensor and good stability over a period of 270 days with minimum aging effects.

Keywords: Humidity sensors; Tungsten doped ZnO; Nano-scaled; Sensitivity; Hysteresis.

\section{INTRODUCTION}

Rapid advancement and automation of technology require humidity sensors for thorough management of ambient moisture in several industrial, medical, pharmaceutical, food packaging and cryogenic processes [1]. Humidity sensors offer monitoring of ambient moisture by converting the vapor content in the atmosphere into electrical signals via transducers. Resistive type nano-scaled metal-oxides belong to class of ceramic humidity sensors. These relative humidity (RH) type metal oxide sensors have attracted much of investigations on account of being light weight, economical, flexible, compact size, durable, high throughput, easy detection and replacement of faults, thermal and long-term stability $[2,3]$. Humidity sensing mechanism is mostly a surface phenomenon which depends upon the interaction of water molecule present in the sensing environment with the surface of the semiconductor metal oxide and is greatly influenced by the presence of nanostructures, inter and intra-granular pores, grain size, pores distribution, grain boundaries, specific surface area and poly-crystals in sensing element [4]. Nano-scaled semiconductor metal oxides find potential applications in nano-devices owing to their increased specific surface area responsible for enhancing surface reactivity. The quantum 
confinement effect is predominant at nano scale which contributes to high efficiency of these nano structures. $\mathrm{ZnO}$ is an extensively explored material for resourceful industrial and miscellaneous applications. Its remarkable properties like direct bad gap of $3.37 \mathrm{eV}$ makes it suitable choice for opto-electronic devices, photo diodes, optical modulator wave guide, solar cells, fluorescence, high luminous transmittance and other electronic applications, as desired conduction can be achieved through doping $[5,6,7,8]$. Further, $\mathrm{ZnO}$ exhibit properties like high refractive index, appreciable thermal, chemical and radiation stability in plasma [9], high exciton binding energy (60 MeV), non-toxicity [10], good substrate adherence, good optical characteristics in plasma [11], high electron production efficiency [12]. $\mathrm{ZnO}$ nano-structures yield high performance FETs, strong room temperature luminescence [13], gas sensors [14] and catalyzers $[15,16]$. Investigating studies led by Borse et al.[17] focusing on the humidity sensing properties of $\mathrm{SnO} 2$ based sensors fired at temperatures $580{ }^{\circ} \mathrm{C}, 680^{\circ} \mathrm{C}, 780^{\circ} \mathrm{C}$ have established that the sensitivity to humidity is highest for $780^{\circ} \mathrm{C}$ firing temperature, observing response time of $140 \mathrm{sec}, 80 \mathrm{sec}, 75 \mathrm{sec}$ and recovery time of $2 \mathrm{~min}, 10 \mathrm{~min}$ and $3 \mathrm{~min}$ for sensors fired at $580^{\circ} \mathrm{C}, 680^{\circ} \mathrm{C}, 780^{\circ} \mathrm{C}$, respectively. The maximum average sensitivity of 5.85 is displayed by the $\mathrm{SnO} 2$ thick film humidity sensor fired at temperature $780^{\circ} \mathrm{C}$. Saidi et al. [18] analyzed moisture sensing characteristics of $\mathrm{Al}$ doped $\mathrm{ZnO}$ film and nano-rods quoting response time to be $555.5 \mathrm{sec}$ and $400 \mathrm{sec}$ respectively. Recovery time of $609 \mathrm{sec}$ for $\mathrm{Al}-\mathrm{ZnO}$ film and $548 \mathrm{sec}$ nanorods were observed; sensitivity being 7.38 and 3.55 respectively for both the samples. Gupta et al. have measured the structural, electrical and humidity sensing properties of nickel oxide ( $\mathrm{NiO})$ nanoparticles annealed at three different temperatures of $200{ }^{\circ} \mathrm{C}, 400{ }^{\circ} \mathrm{C}$ and $700{ }^{\circ} \mathrm{C}$ and found that $\mathrm{NiO}$ sample annealed at $700{ }^{\circ} \mathrm{C}$ is having the highest sensing response of $47.09 \%$ at $90 \%$ RH [19]. Misra et al. have demonstrated the sensitivity of $\mathrm{SnO} 2$ doped $\mathrm{ZnO}$ sensor to be 32.16 
$\mathrm{M} \Omega / \% \mathrm{RH}$ for the sample SZ-15 annealed at $700^{\circ} \mathrm{C}$. Crystal morphologies such as grain size, crystallinity, porosity, grain boundaries, induced lattice strains are the characteristics of $\mathrm{ZnO}$ nanostructures [20,21]. Addition of a dopant bring about change in the morphology thereby altering the lattice parameters, mechanical features like lattice strain, stress, electrical, humidity sensing behavior and invoking new functionalities. This endeavor reports dopant induced changes in structural, peak broadening, morphological and humidity sensing response for progressive incorporation of tungsten in different molar ratios ( $\mathrm{x}=0,0.50,1.25,1.75 \mathrm{~mol} \%)$.

\section{Material and Methods}

\subsection{Nanoparticle Synthesis}

The precursors include Zinc acetate di-hydrate $\left[\mathrm{Zn}\left(\mathrm{CH}_{3} \mathrm{COO}\right)_{2} .2 \mathrm{H}_{2} \mathrm{O}, 99.99 \%\right.$ pure Loba Chemie], Sodium tungstate di-hydrate $\left[\mathrm{Na}_{2} \mathrm{WO}_{4} \cdot 2 \mathrm{H}_{2} \mathrm{O}, 99.99 \%\right.$ pure Loba Chemie], Sodium hydroxide $[\mathrm{NaOH}]$ and ethanol. The $\mathrm{W}-\mathrm{ZnO}$ nanoparticles are synthesized through coprecipitation method. Initially, a solution of $0.1 \mathrm{M}$ zinc acetate is prepared in $50 \mathrm{ml}$ of ethanol. This solution is refluxed at $80^{\circ} \mathrm{C}$ with constant stirring in a magnetic stirrer. Another solution of $0.1 \mathrm{M}$ sodium tungstate is separately prepared in ethanol. These two solutions are mixed in molar ratios to obtain pure, $0.50 \mathrm{~mol} \%, 1.25 \mathrm{~mol} \%$ and $1.75 \mathrm{~mol} \%$ tungsten doped $\mathrm{ZnO}$. The obtained solutions are vigoursly stirred in a magnetic stirrer for 5 hours at a temperature of $80^{\circ} \mathrm{C}$ to form a homogeneous mixture. Consequently, $0.1 \mathrm{M}$ sodium hydroxide solution is added drop wise to gradually increase the $\mathrm{pH}$ of the solution to 11 . After the addition of $\mathrm{NaOH}$ the metal ions start to co-precipitate in the form of metal hydroxide thereby initiating nuclei formation. The precipitate obtained is filtered and washed 2-3 times in ethanol. The washed precipitates are dried at $150^{\circ} \mathrm{C}$ and grounded into fine powder. Finally, the obtained powder is calcined for 6 hours at $500^{\circ} \mathrm{C}$ to 
yield powders of required concentrations of tungsten in $\mathrm{ZnO}$. Fig.1 represents schematic synthesis of the pure and tungsten doped $\mathrm{ZnO}$.

\subsection{Preparation of Nano- humidity sensors}

The nano-sensor samples are prepared in the form of pellets as pelletized samples tend to possess high surface uniformity and elevated pore density throughout the surface of the pellet [22]. The pelletized humidity nano-sensors are obtained by mixing polyvinyl alcohol (10\% weight) with the calcined powder as binder. Binder is used to increase the strength of the sample making it consolidate. Mixed powder is then grounded using mortar and pestle to uniformity. The nano-sensors are pelletized under uniaxial isostatic compression of $350 \mathrm{MPa}$ in a hydraulic press machine (M.B. Instruments, Delhi, India) at room temperature. The prepared pellet samples are discs of diameter $10 \mathrm{~mm}$ and $2 \mathrm{~mm}$ in thickness. Finally, pressed powder pellets are sintered at $500^{\circ} \mathrm{C}$ for 5 hours in an electric muffle furnace (Ambassador, India) and gradually cooled to room temperature.

\section{Results and discussion}

\subsection{Morphological and structural analysis}

The phase identification and crystal growth orientation of prepared nanostructures are characterized by High-Definition X-ray Diffractometer. The recorded diffraction spectra for pure and doped $\mathrm{ZnO}_{1-\mathrm{x}} \mathrm{W}_{\mathrm{x}}(\mathrm{x}=0,0.50,1.25,1.75 \mathrm{~mol} \%)$ samples are depicted in Fig.2a. The diffraction peaks are prominent and intense, the strongest peak corresponds to the plane (101) for all the prepared samples. All the well indexed peaks are consistent with hexagonal wurtzite structure of $\mathrm{ZnO}$ (JCPDS card 01-073-8765). Absence of any detectable, dopant related 
peak suggests that tungsten ions are successfully incorporated into the zinc oxide lattice thereby rendering the original wurtzite structure of zinc oxide ruling out the presence of any impurity. The prepared nanostructures are crystalline in nature and indicates no phase transition and exist in single phase. The microstructural parameters like crystallite size and micro-strain in the crystal lattice may be obtained by various techniques namely Scherrer's Method (SM), Williamson-Hall Method (WHM) to measure crystallite size and size-strain plot Method (SSPM) to study the strain effect on the crystal lattice.

\subsection{Deby-Scherrer's Method:}

The average crystallite size of all the nano-structured samples can be calculated using Deby-Scherrer's expression;

$$
D=\frac{\kappa \lambda}{\beta_{D} \sin \theta}
$$

Where, $\mathrm{D}$ is the average crystallite size, $\kappa=0.9$ (dimensionless) is the shape factor, $\lambda=1.5406 \AA$ is the wavelength of Cuka line, $\theta$ is the measured Braggs' diffraction angle and $\beta_{\mathrm{D}}$ the full width at half maxima intensity (FWHM) in radians.

The obtained broadening $\beta_{\mathrm{D}}$ is the convolution of the broadening due to instrument as well as sample related broadening [23] related as;

$$
\beta_{\mathrm{D}}^{2}=\beta^{2} \text { instrumental }+\beta_{\text {sample }}^{2}
$$




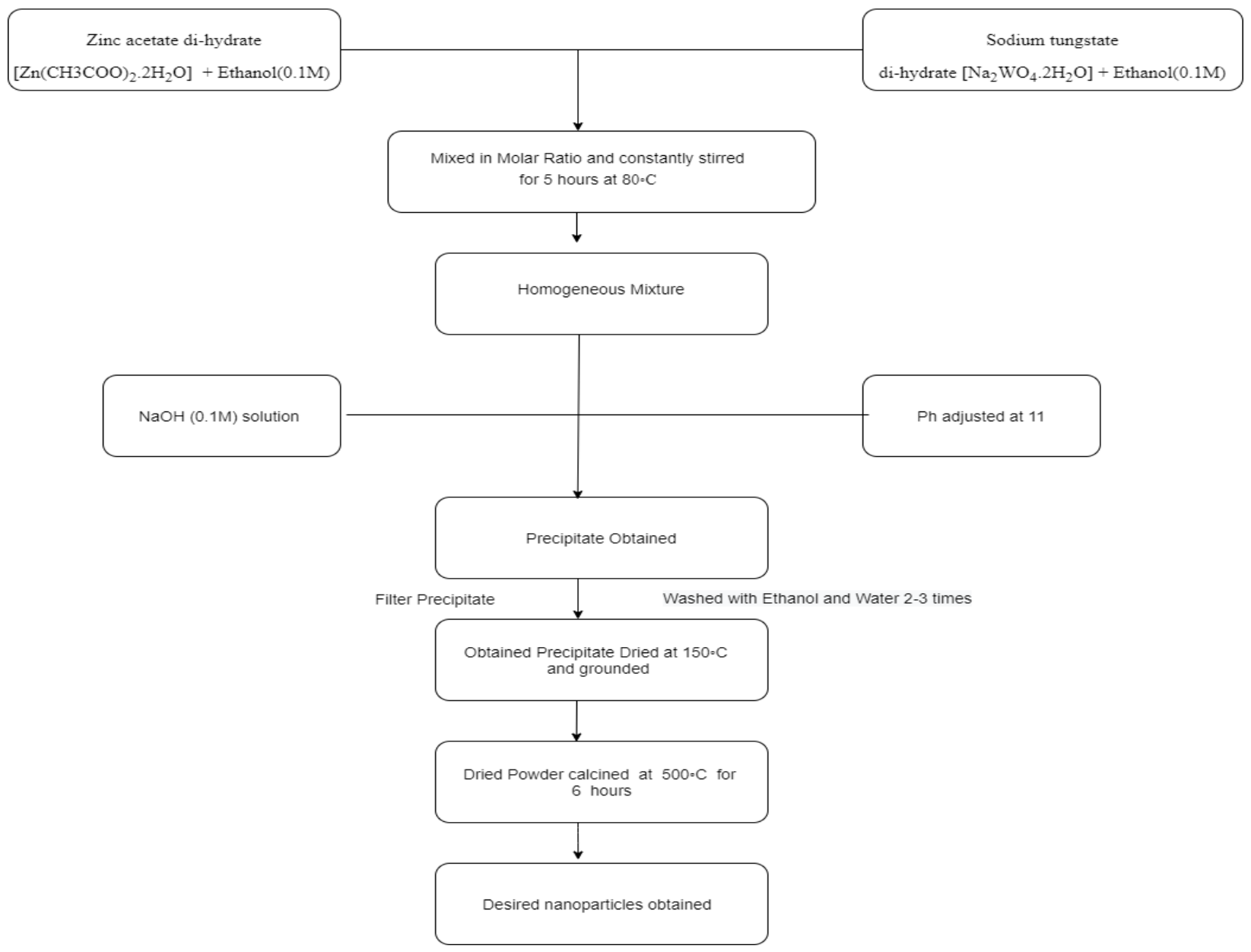

Fig. 1 The schematic represntation co-precipitated $\mathrm{ZnO}$ nano particles. 


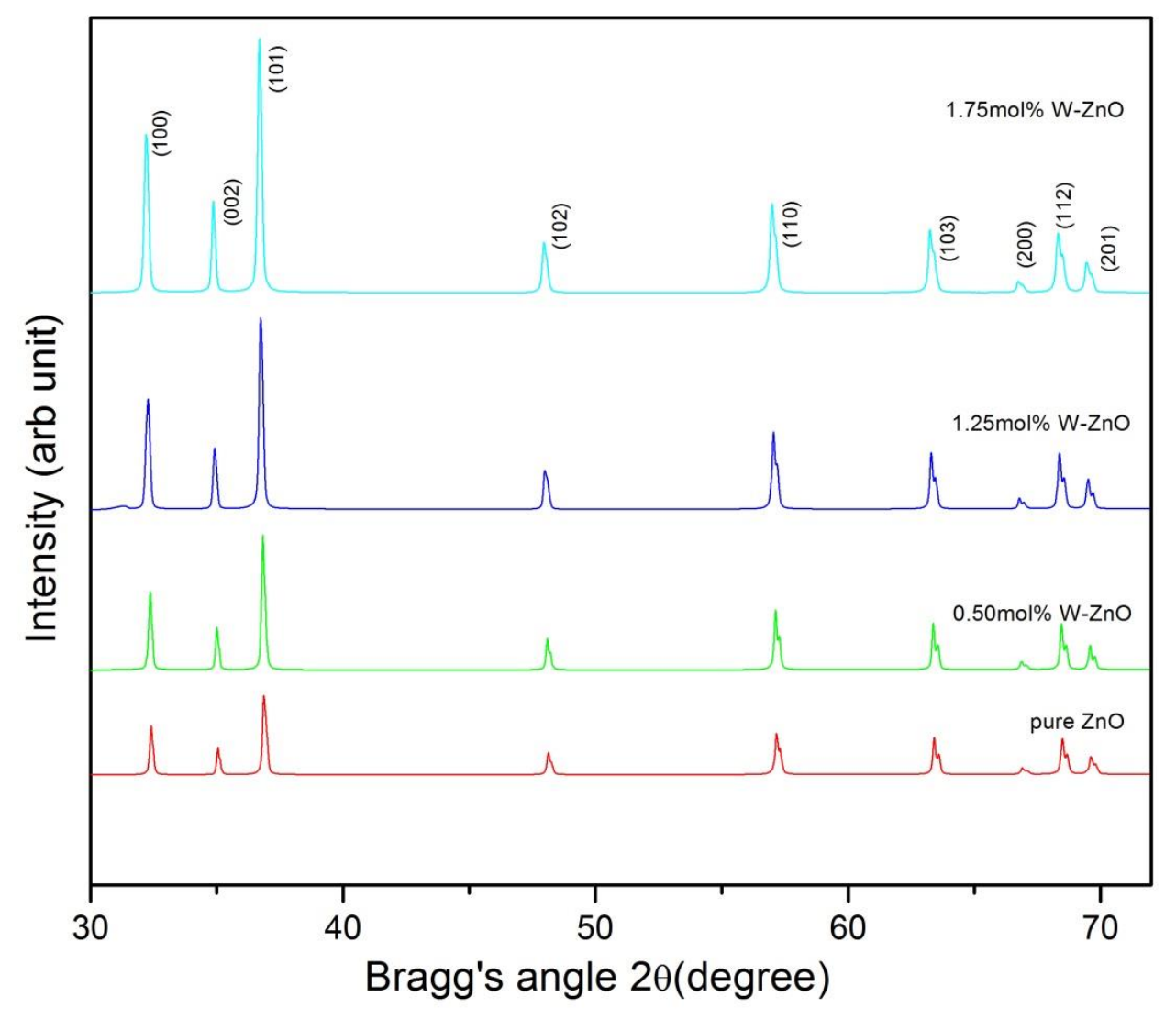

Fig.2a. XRD spectra of pure and $\mathrm{W}-\mathrm{ZnO}$. 


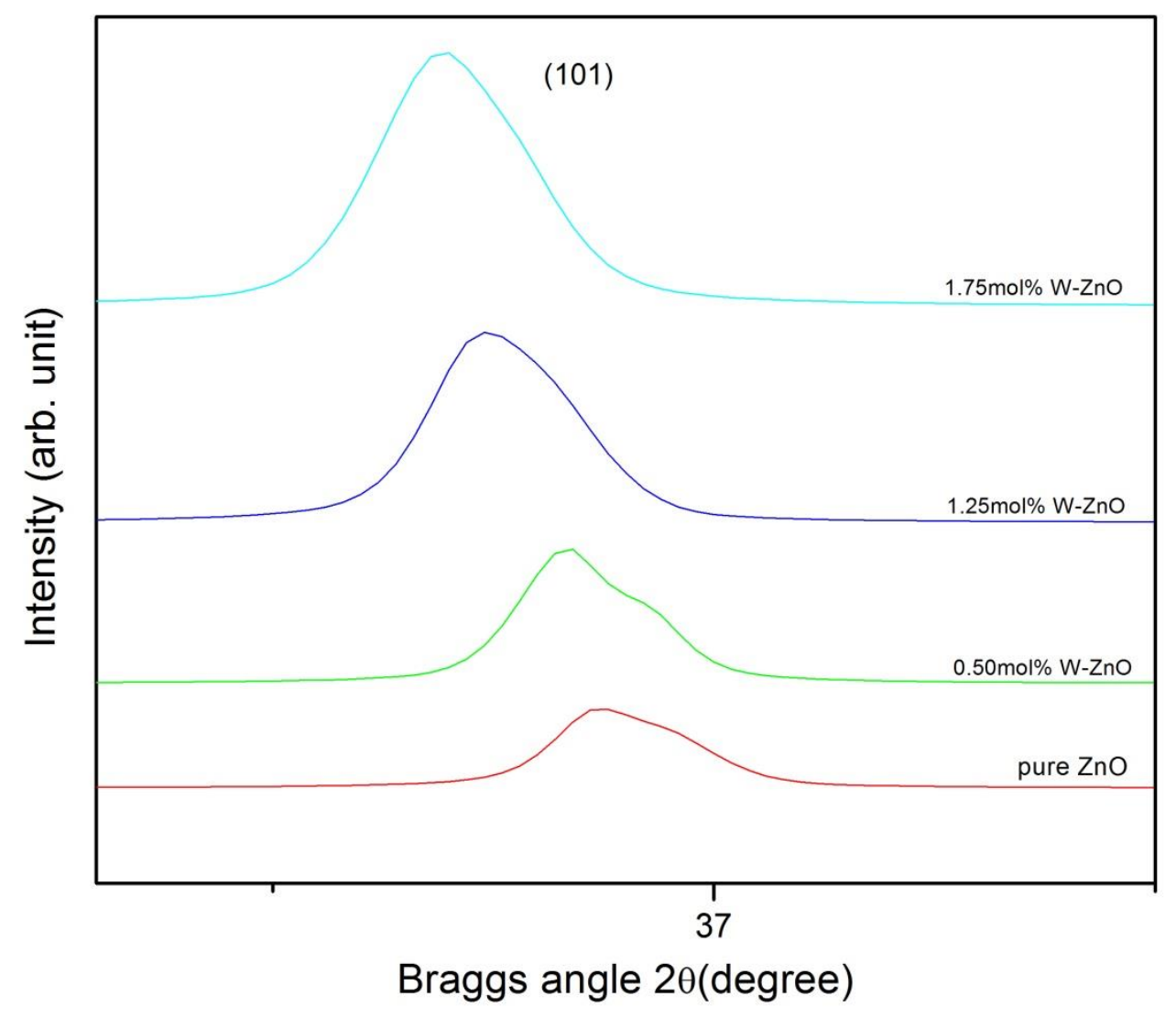

Fig.2b XRD peak corresponding to plane (101) for $\mathrm{W}$ - ZnO samples. 
Lattice parameters $\mathrm{a}=\mathrm{b}$ and $\mathrm{c}$ for hexagonal wurtzite structure of pure and doped $\mathrm{ZnO}$ samples corresponding to (h,k,l) planes (100) and (101) are estimated from Bragg's equations (3), (4), (5) and (6) and the volume of the unit cell is determined from equation (7).

$$
\begin{gathered}
2 \mathrm{~d} \sin \theta=\mathrm{n} \lambda \\
\frac{1}{d^{2}}=\frac{4}{3}\left(\frac{h^{2}+h k+k^{2}}{a^{2}}\right)+\frac{l^{2}}{c^{2}} \\
a=\frac{\lambda}{\sqrt{3} \sin \theta}\left(\sqrt{h^{2}+h k+k^{2}}\right) \\
c=\frac{\lambda}{2 \sin \theta} l \\
V=\frac{\sqrt{3} a^{2} c}{2}=0.866 a^{2} c
\end{gathered}
$$

The lattice parameters as determined through HDXRD data using the above equations for hexagonal wurtzite are $\mathrm{a}=\mathrm{b}=0.2759 \mathrm{~nm}$ and $\mathrm{c}=0.5175 \mathrm{~nm}$ for crystal axes $\alpha=\beta=90^{\circ}$ and $\gamma=120$. These values are in good agreement with the lattice parameters of the bulk $\mathrm{ZnO}$ [24]. Fig.2b shows that the dominant XRD peak corresponding to (101) plane shift to lower diffraction angles of $2 \theta$ as the doping escalates and average crystallite size of pure and $\mathrm{W}^{6+}$ doped $\mathrm{ZnO}$ sample decreases from $44.46 \mathrm{~nm}$ to $37.68 \mathrm{~nm}$. The crystallite size appears to decrease accompanied by broadening of FWHM, with escalation of dopant incorporation from 0 to $1.75 \mathrm{~mol} \%$ of $\mathrm{ZnO}$. This decrement in the crystallite size as calculated using Deby-Scherrer's formula which may be attributed to the replacement of $\mathrm{Zn}^{2+}[0.074 \mathrm{~nm}]$ ions by $\mathrm{W}^{6+}[0.062 \mathrm{~nm}]$ ions during the 
nucleation of the crystal sites, $\mathrm{W}^{6+}$ being of smaller radii, occupy the interstitial sites of $\mathrm{Zn}^{2+}$ ion and thereby inhibit proper crystal growth in preferable orientation [25]. An indication of $\mathrm{W}^{6+}$ incorporation into the crystal lattice of $\mathrm{ZnO}$ can be concluded from the shifting of $\mathrm{XRD}$ peaks towards lower diffraction angles (Fig.2b) which may cause an expansion of $\mathrm{ZnO}$ crystal lattice and an increase in the interplanar distance ' $d$ ' [26]. The expansion of lattice due to tungsten incorporation can be justified with the increased values of lattice parameters as compared to pure $\mathrm{ZnO}$. This can also supported by the fact that the most prominent peak in the diffraction spectra shifts towards the lower diffraction angles as the concentration is increased from pure to 1.75 mol\% of $\mathrm{W}^{6+}$ doping in $\mathrm{ZnO}$. Further, this reduction in the crystallite size with increase in doping percentage may have resulted from inhibited crystal growth due to compressive stress, as the increase in doping concentration may have caused a deterioration of lattice constant. The wurtzite structure is composed of tetrahedrally co-ordinated $\mathrm{O}^{2-}$ and $\mathrm{Zn}^{2+}$ ions arranged in alternating planes stacked along the c-axis. $\mathrm{ZnO}$ has a strong tendency to grow along c-axis because of higher energy of this plane rather than the planes oriented along other directions [27].

Table 1. Structural parameters of undoped and $\mathrm{W}$ doped $\mathrm{ZnO}$ nano-structures.

\begin{tabular}{|c|c|c|c|c|c|c|}
\hline \multicolumn{7}{|c|}{ Deby-Scherrer's Method } \\
\hline Samples & $\begin{array}{l}\text { Bragg's } \\
\text { angle }\end{array}$ & $\begin{array}{l}\text { Crystallite } \\
\text { Size }(n m)\end{array}$ & $\begin{array}{l}\text { Interplanar } \\
\text { Spacing (̊̊) }\end{array}$ & Lattice $\mathbf{C}$ & $\operatorname{tant}(\AA)$ & $\begin{array}{l}\text { Unit Cell } \\
\text { Volume }\end{array}$ \\
\hline $\begin{array}{l}\text { (Doping } \\
\text { mol\%) }\end{array}$ & $2 \theta$ (deg.) & $\mathbf{D}$ & d & $\mathbf{a}=\mathbf{b}$ & $\mathbf{C}$ & $\mathbf{V}$ \\
\hline 0 & 36.864 & 44.46 & 2.435330 & 3.209084 & 5.1127 & 45.59637 \\
\hline 0.50 & 36.829 & 41.14 & 2.437564 & 3.212152 & 5.1212 & 45.75955 \\
\hline 1.25 & 36.741 & 40.09 & 2.443200 & 3.221296 & 5.1326 & 46.12290 \\
\hline 1.75 & 36.695 & 37.68 & 2.446157 & 3.227439 & 5.1412 & 46.37654 \\
\hline
\end{tabular}




\section{Williamson-Hall Analysis}

\subsection{Uniform Deformation Model (UDM)}

W-H analysis is employed to study peak-broadening, calculate the crystallite size, structural parameters, micro-strains and phase composition. The crystallite size calculated by W$\mathrm{H}$ analysis is more accurate [28]. Assuming the isotropy of the crystal all the properties remains the same throughout the crystallographic directions. Micro-strains arising within the crystals are due to their finite size. The peak broadening indicates refinement associated with strain induced due to crystal imperfections and dislocations is expressed in the terms of strain in the crystal lattice " $\varepsilon$ " strain induced broadening " $\beta$ " in equation (8);

$$
\varepsilon=\frac{\beta \varepsilon}{4 \tan \theta}
$$

The net peak broadening is the combined effect of peak broadening due to crystallite size (Scherrer's broadening) $\beta_{\mathrm{D}}$ and strain induced broadening $\beta_{\varepsilon}$.

$$
\beta_{\mathrm{hkl}}=\beta_{\mathrm{D}}+\beta_{\varepsilon}
$$

$\beta_{\mathrm{hkl}}$ being the instrument corrected full-width half maximum. Both crystallite size and strain show different variation with respect to the Bragg's angle which dictate the dependence of peak broadening on crystallite size and micro-strain [29]. Therefore, equation (9) evolves to;

$$
\beta_{\mathrm{hk}}=\frac{\kappa \lambda}{D \cos \theta}+4 \varepsilon \tan \theta
$$

The above equation is the Williamson-Hall equation which can also be depicted as; 


$$
\beta_{h k l} \cos \theta=\frac{K \lambda}{D}+4 \varepsilon \sin \theta
$$

A graph (fig.3) plotted for $4 \sin \theta$ on the $\mathrm{x}$-axis against $\beta_{\mathrm{hk} k \mathrm{cos}} \theta$ on the $\mathrm{y}$-axis. From linear fit to data, the crystallite size is estimated from the y-intercept $\left(K \lambda D_{W-H}\right)$ and strain from the slope of the fit between $4 \sin \theta$ and $\beta_{\mathrm{hkl}} \cos \theta$. The compressive strain induced by lattice shrinkage in the crystals, is obvious from the lattice parameters [30]. The reduction in the crystallite size as per increase in doping concentration causes increase in the specific surface are of the nanostructures.

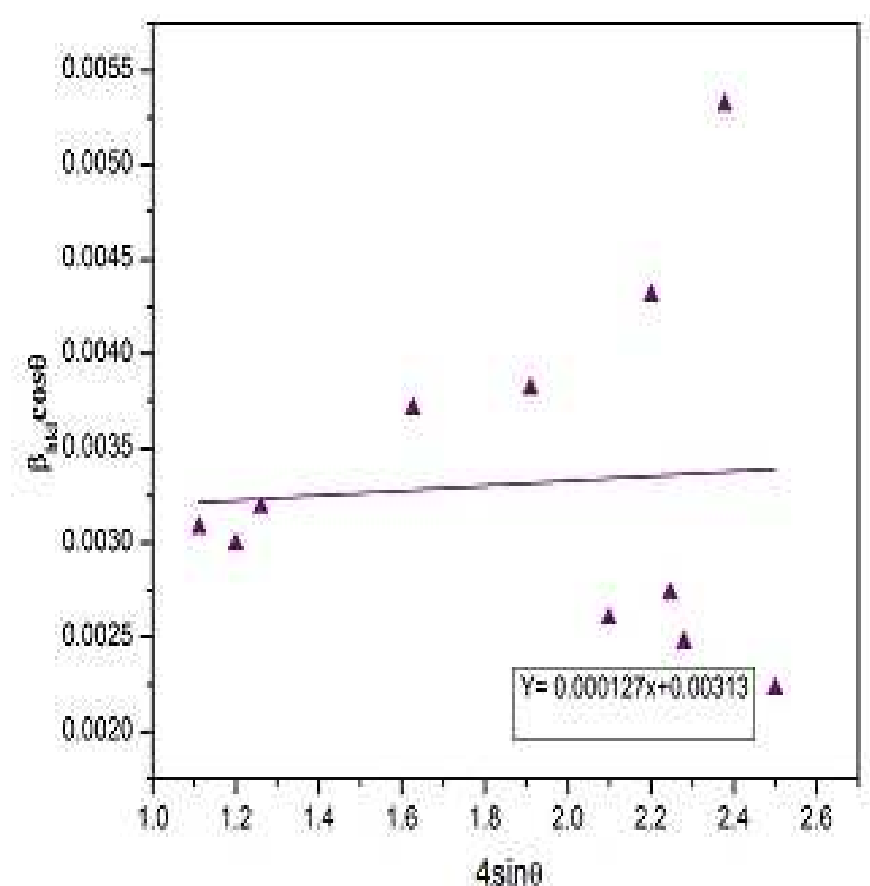

(a)

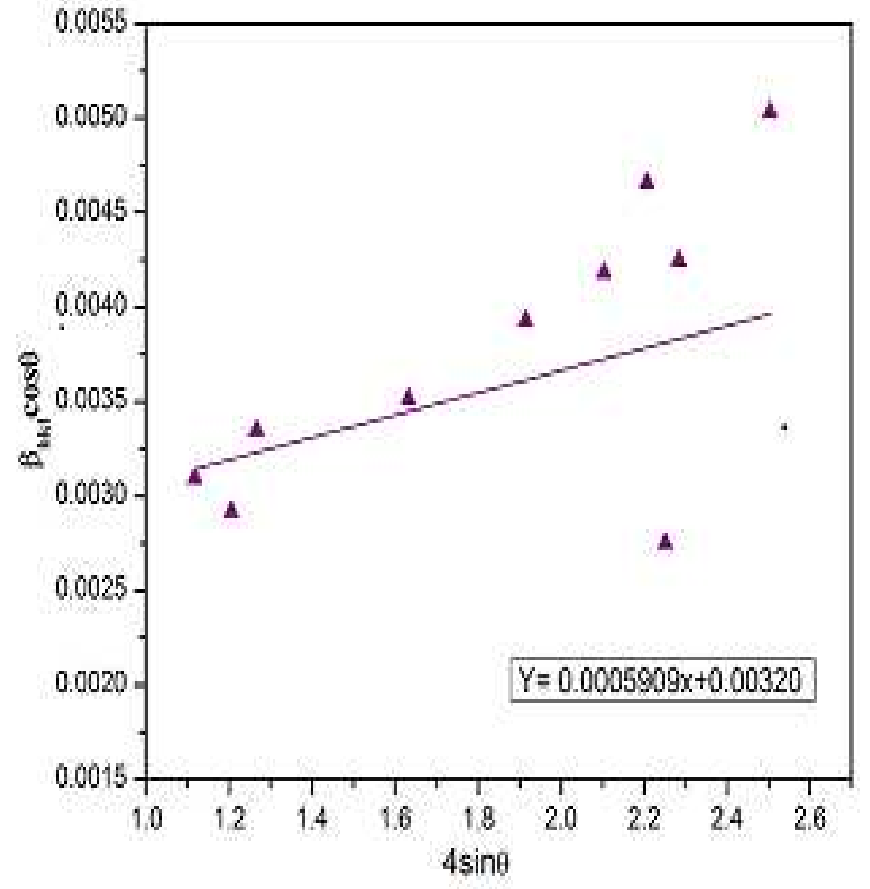

(b) 


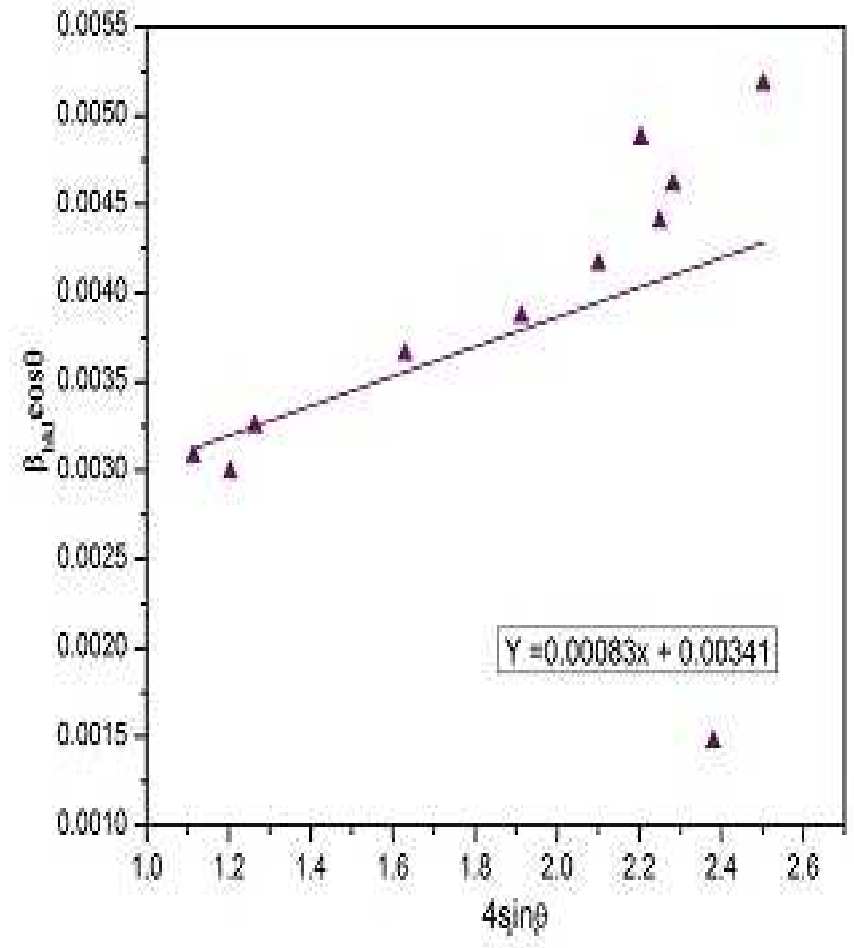

(c)

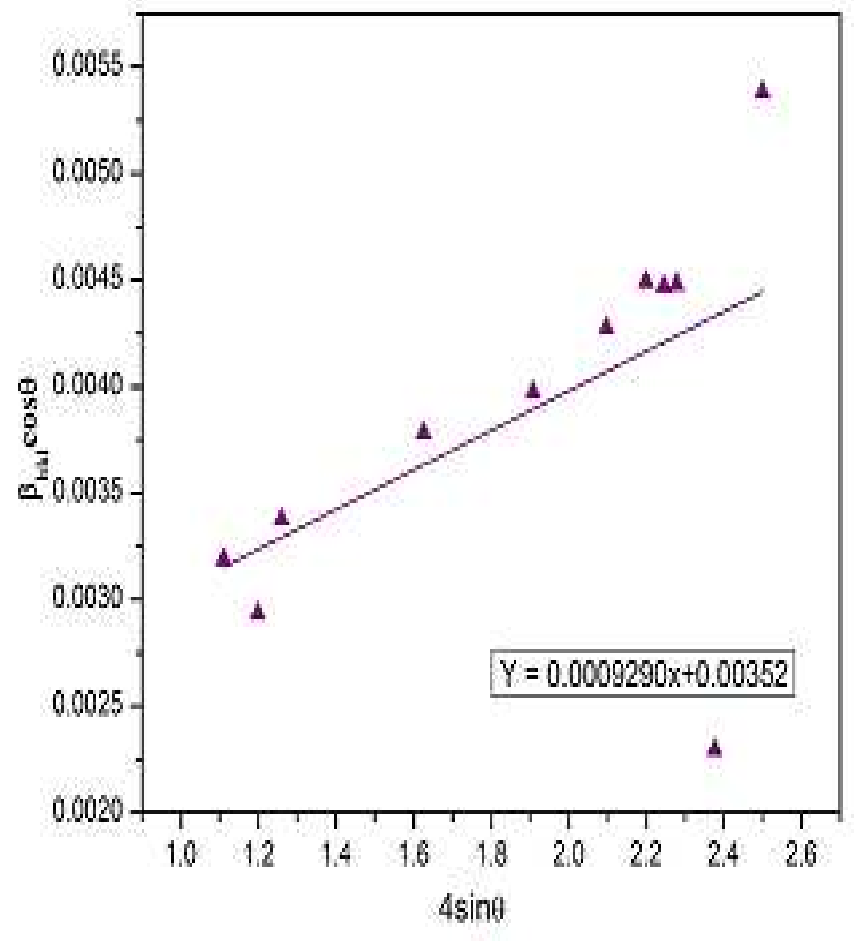

(d)

Fig. 3 The W-H Analysis of undoped and doped ZnO Nano particles considering UDM

(a) UDM for pure $\mathrm{ZnO}$ (b) UDM of $0.50 \mathrm{~mol} \% \mathrm{~W}-\mathrm{ZnO}$ (c) UDM of $1.25 \mathrm{~mol} \% \mathrm{~W}-\mathrm{ZnO}$

(d) UDM of $1.75 \mathrm{~mol} \% \mathrm{~W}-\mathrm{ZnO}$.

\subsection{Uniform Stress Deformation Model (USDM)}


For small values of strain the Hook's law implies a linear relation between strain and stress as $\sigma=\mathrm{Y} \epsilon, \sigma$ being stress, $\mathrm{Y}$ is the Young modulus and $\epsilon$ is the strain. This model takes into account the anisotropy of Hook's law in the crystal. Equation (11) can now be modified accordingly as below;

$$
\beta_{h k l} \cos \theta=\frac{K \lambda}{D}+\frac{4 \sigma \sin \theta}{Y_{h k l}}
$$

The stress in the pure and $\mathrm{W}$ doped $\mathrm{ZnO}$ samples can be calculated from the graph (Fig.4) of $\beta_{\mathrm{hkl}} \cos \theta$ verses $4 \sin \theta / \mathrm{Y}_{\mathrm{hkl}}$, where for the linear fit of data, slope will represent the strain $(\epsilon)$ and

Crystallite size can be obtained from the intercept, and stress can be deduced from strain. The Young's modulus for a hexagonal structure is calculated from expression (13);

$$
Y=\frac{\left[h^{2}+\frac{(h+2 k)^{3}}{3}+\left(\frac{a l}{c}\right)^{2}\right]^{2}}{s_{11}\left(h^{2}+\frac{(h+2 k)^{2}}{3}\right)^{2}+s_{33}\left(\frac{a l}{c}\right)^{4}+\left(2 s_{13}+s_{44}\right)\left(h^{2}+\frac{(h+2 k)^{2}}{3}\right)\left(\frac{a l}{c}\right)^{2}}
$$

Where $\mathrm{s}_{11}, \mathrm{~s}_{13}, \mathrm{~s}_{33}, \mathrm{~s}_{44}$ are referred as elastic compliances of $\mathrm{ZnO}$ having values $7.858 \times 10^{-12}$,$2.206 \times 10^{-12}, 6.940 \times 10^{-12}, 23.57 \times 10^{-12} \mathrm{~m}^{2} \mathrm{~N}^{-1}$ respectively [31]. 


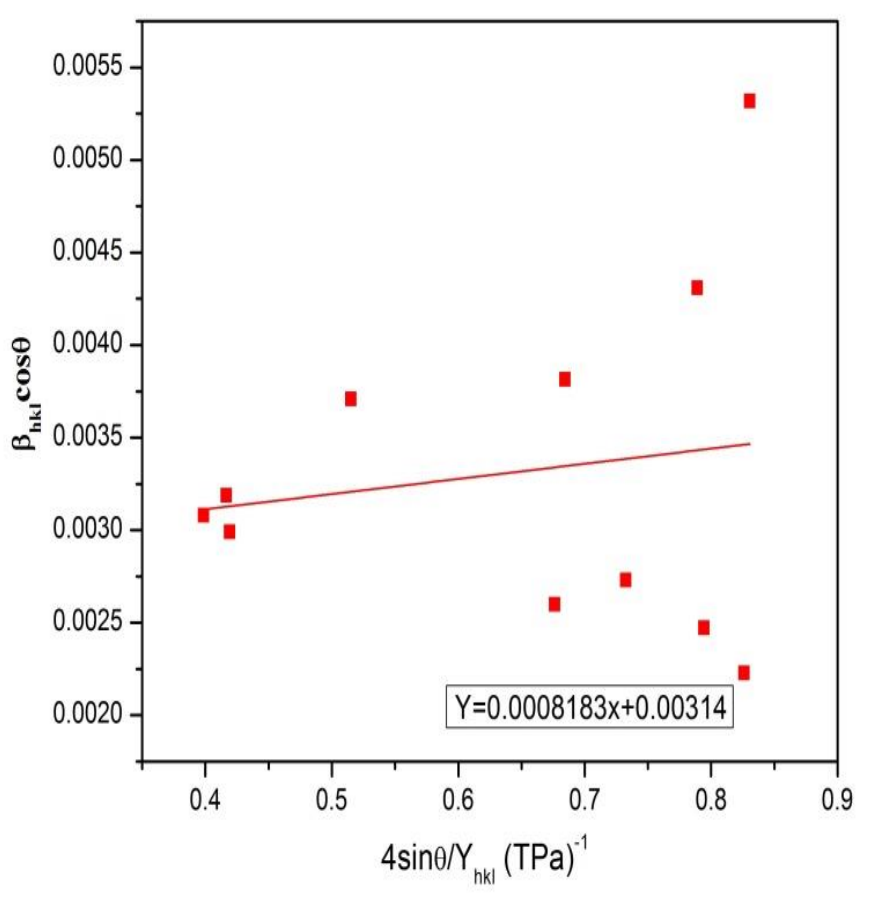

(a)

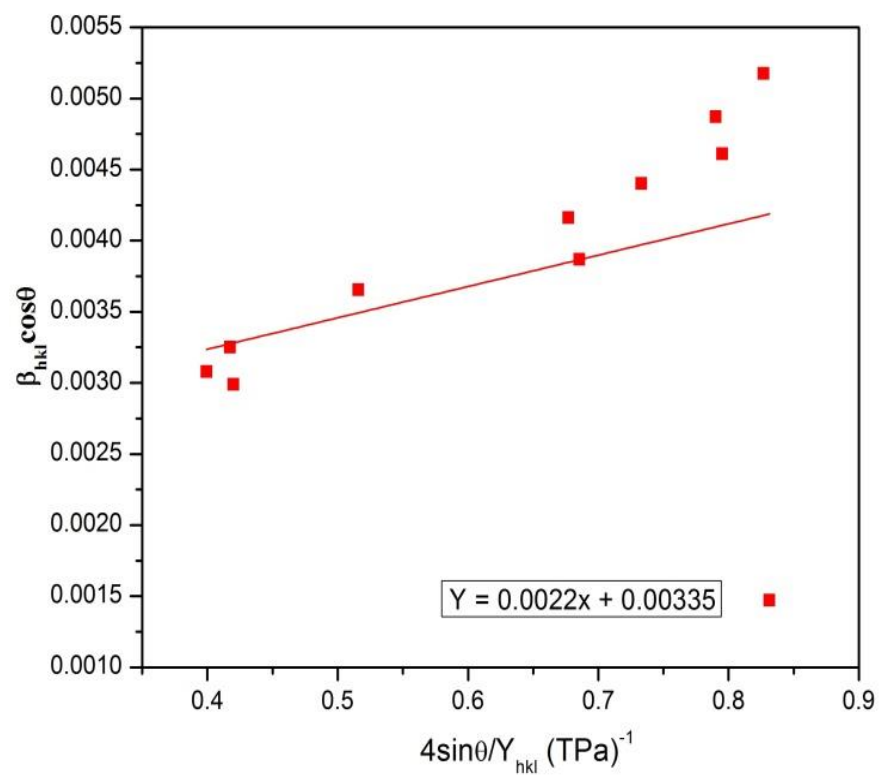

(c)

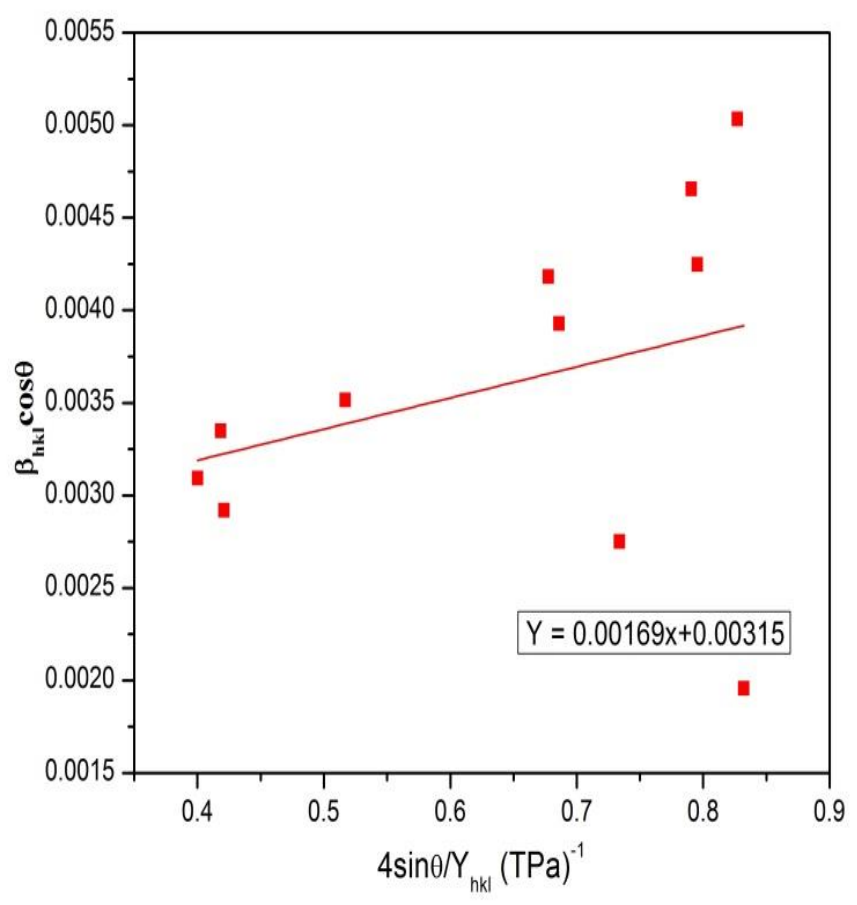

(b)

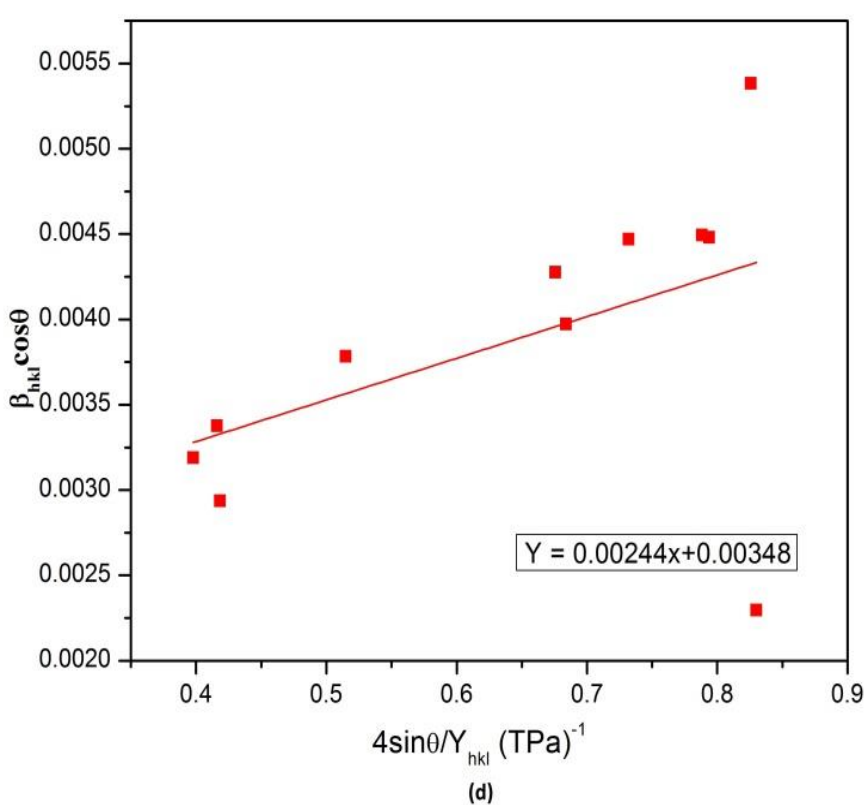


Fig.4. Remodeled W-H Analysis of undoped and doped ZnO Nano particles considering USDM. (a)USDM of pure $\mathrm{ZnO}$ (b) USDM of $0.50 \mathrm{~mol} \% \mathrm{~W}-\mathrm{ZnO}$ (c)USDM of $1.25 \mathrm{~mol} \% \mathrm{~W}-\mathrm{ZnO}$ (d)USDM of $1.75 \mathrm{~mol} \% \mathrm{~W}-\mathrm{ZnO}$.

\subsection{Uniform Energy Density Model (UDEDM)}

The energy density which is energy per unit volume of a nano-crystal is not isotropic. And the constant for Young modulus is no more a constant relating strain and stress of the system therefore we have to consider inhomogeneity and anisotropy while calculating the energy density of the material. For UDEDM, according to Hook's law, the energy density varies as a function of strain as

$$
u=\frac{\varepsilon^{2} Y_{h k l}}{2}
$$

The equation (14) modifies equation (11) such that;

$$
\beta_{h k l} \cos \theta=\frac{K \lambda}{D}+4\left(\frac{2 u}{Y_{h k l}}\right)^{2} \sin \theta
$$

Fig. 5 represents remodeled $\mathrm{W}-\mathrm{H}$ Analysis of undoped and doped $\mathrm{ZnO}$ Nano particles considering UDEDM. The slope and the intercept of linear fit to data plot between $\left(2 \mathrm{u} / \mathrm{Y}_{\mathrm{hkl}}\right)^{2}$ on $\mathrm{x}$-axis and of $\beta_{\mathrm{hkl}} \cos \theta \quad \mathrm{y}$-axis gives the uniform energy density (u) and crystallite size respectively. Stress $\sigma$ can be evaluated by the knowledge of $\mathrm{Y}_{\mathrm{hkl}}, \mathrm{u}$ and $\epsilon$ from relation $\sigma=\mathrm{Y}_{\mathrm{hkl}} \epsilon$ and equation (14). 

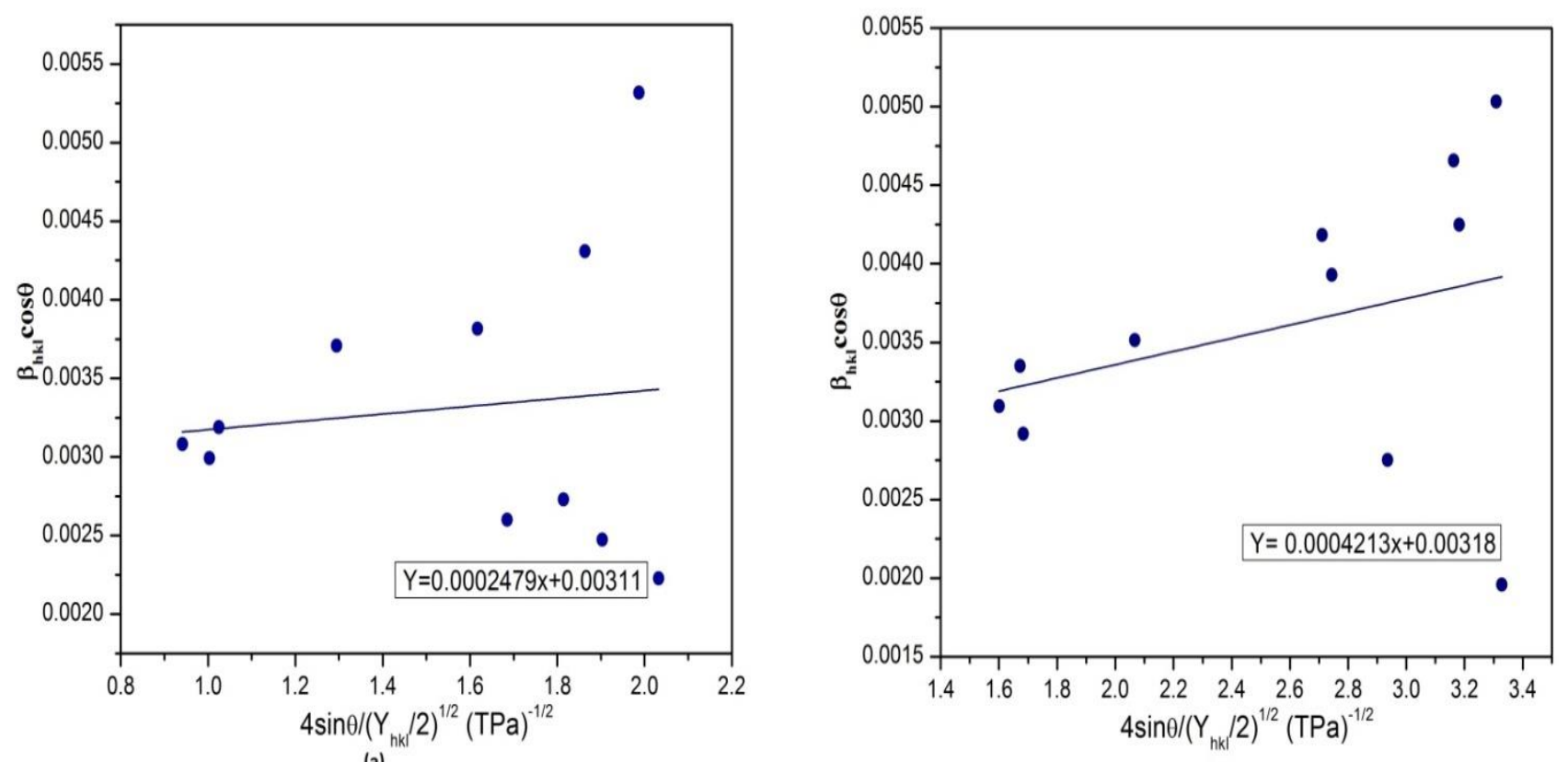

(a)
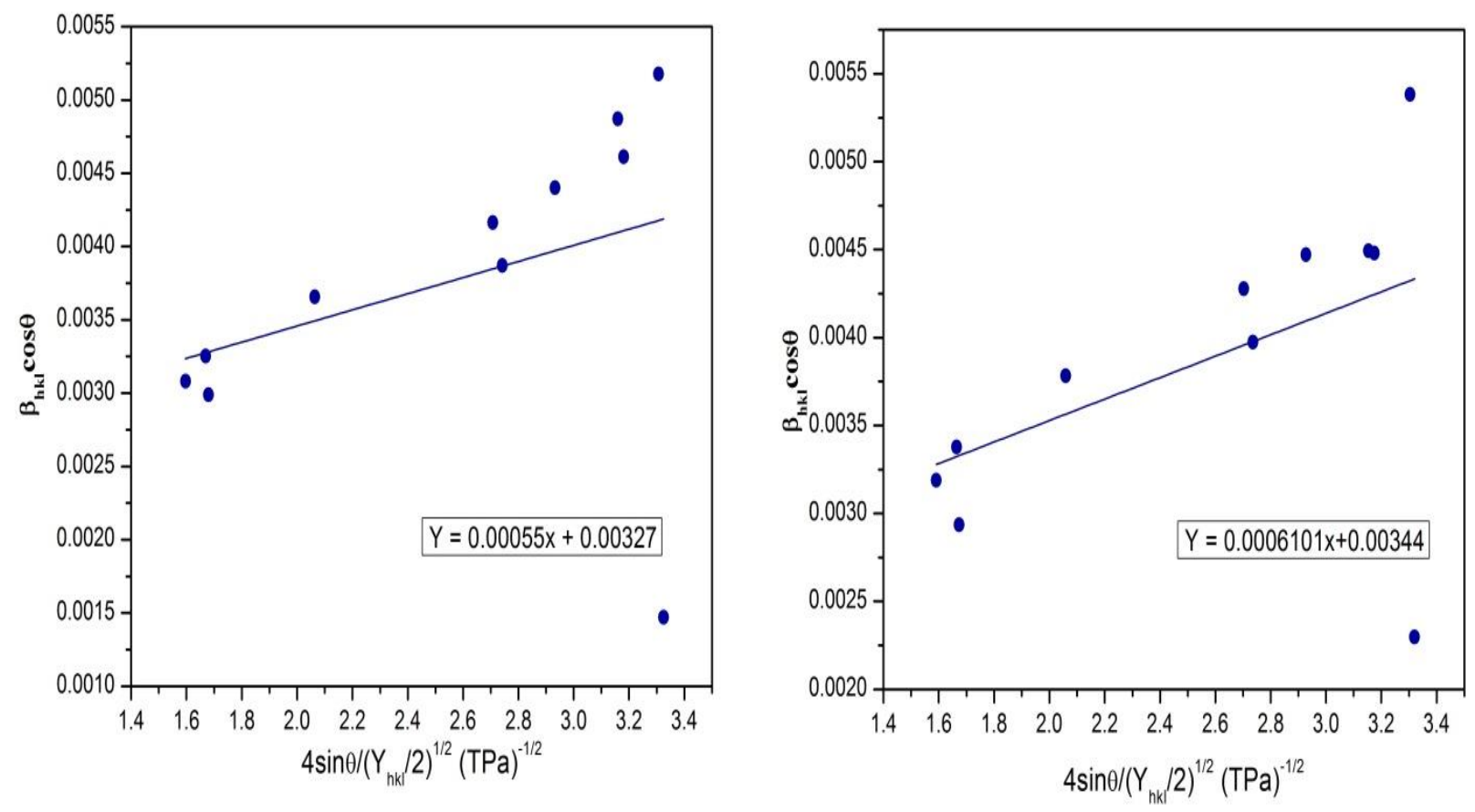

(c)

(d) 
Fig.5 Remodeled W-H Analysis of undoped and doped ZnO Nano particles considering UDEDM (a)UDEDM of pure $\mathrm{W}-\mathrm{ZnO}$ (b)UDEDM of $0.50 \mathrm{~mol} \% \mathrm{~W}-\mathrm{ZnO}$ (c)UDEDM of $1.25 \mathrm{~mol} \% \mathrm{~W}-\mathrm{ZnO}(\mathrm{d}) \mathrm{UDEDM}$ of $1.75 \mathrm{~mol} \% \mathrm{~W}-\mathrm{ZnO}$.

\subsection{Size-strain plot method}

Williamson-Hall method suggests isotropic nature of line broadening. Strain-size plot considers the isotropic nature of diffracting crystal domains rendering to micro-strain effect. Size-strain plot proves an accurate tool to calculate size-strain parameters by eliminating the discrepancies caused at higher diffraction angles. In addition to the isotropic line broadening an average size-strain plot results in precise evaluation of strain-size parameters by investigating only lower diffraction angle [32]. In this approximation the function for "crystallite size" is taken to be Lorentzian and that of the "strain -profile" is "Gaussian function" [33]. The equation for size-strain plot according to above considerations can be concluded as;

$$
\left(d_{h k l} \beta_{h k l} \cos \theta\right)^{2}=\frac{k}{D} d^{2}{ }_{h k l} \beta_{h k l} \cos \theta+\left(\frac{\varepsilon}{2}\right)^{2}
$$

$k$ is a constant having value $4 / 3$ for spherical particles. The size-strain plot is plotted for all orientational peaks corresponding to diffraction angle from $2 \theta=20^{\circ}$ to $2 \theta=80^{\circ} \mathrm{d}^{2}{ }_{\mathrm{hkl}} \beta \beta_{\mathrm{hkl}} \cos \theta$ on $\mathrm{x}$-axis and $\left(\mathrm{d}_{\mathrm{hkl}} \beta \mathrm{hkl} \cos \theta\right)^{2}$ on the $\mathrm{y}$-axis are represented in fig. 6 . The crystallite size is calculated from the slope of the linear fitted curve and strain is the root of the intercept on y-axis. 


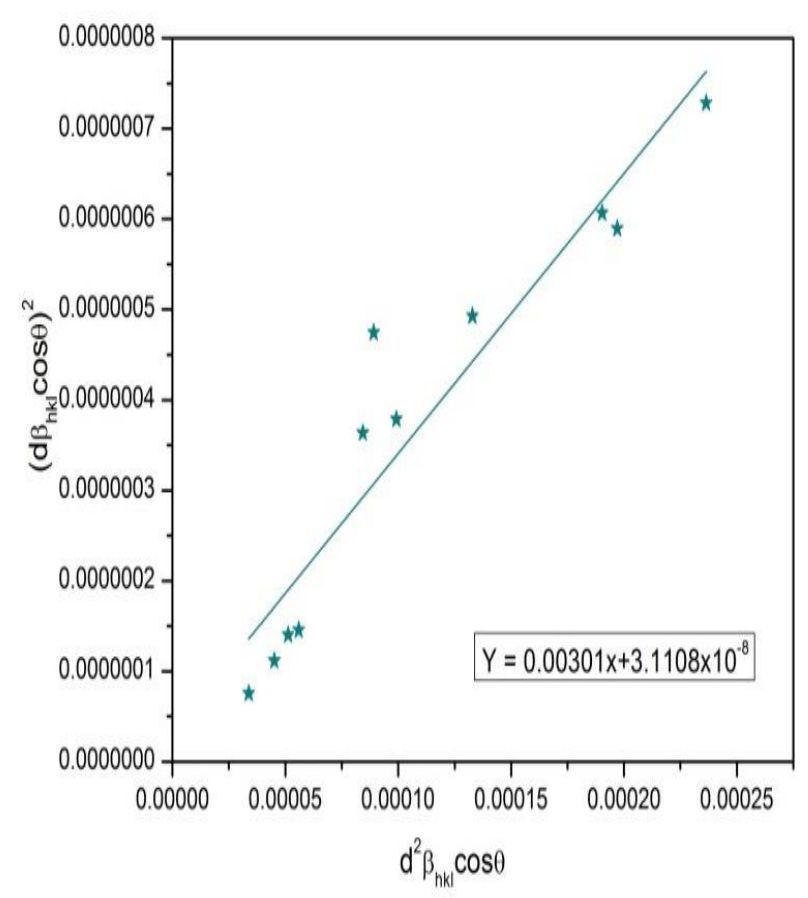

(a)

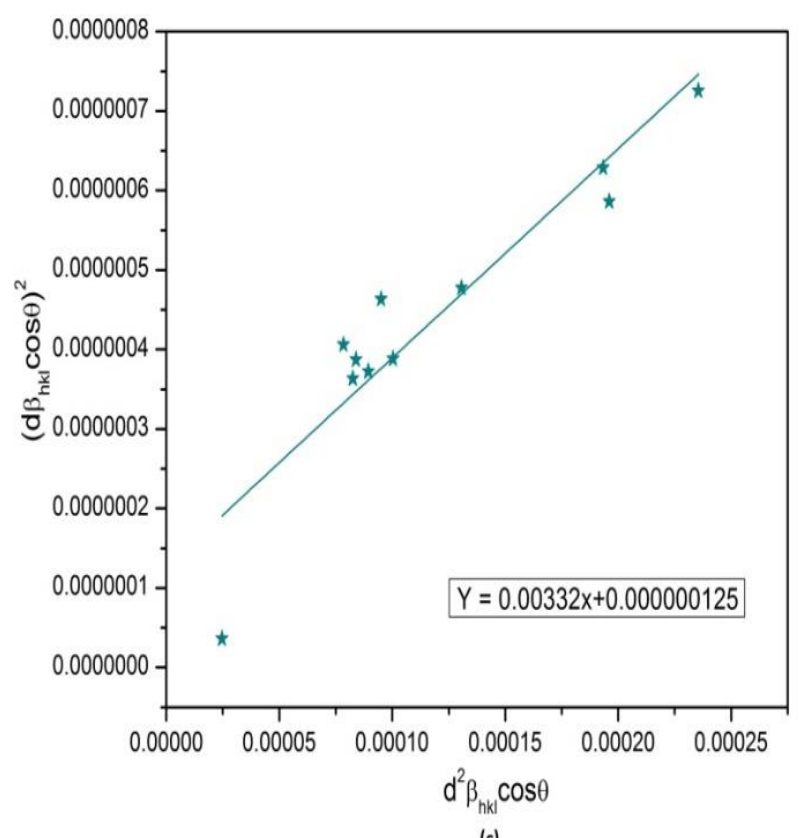

(c)

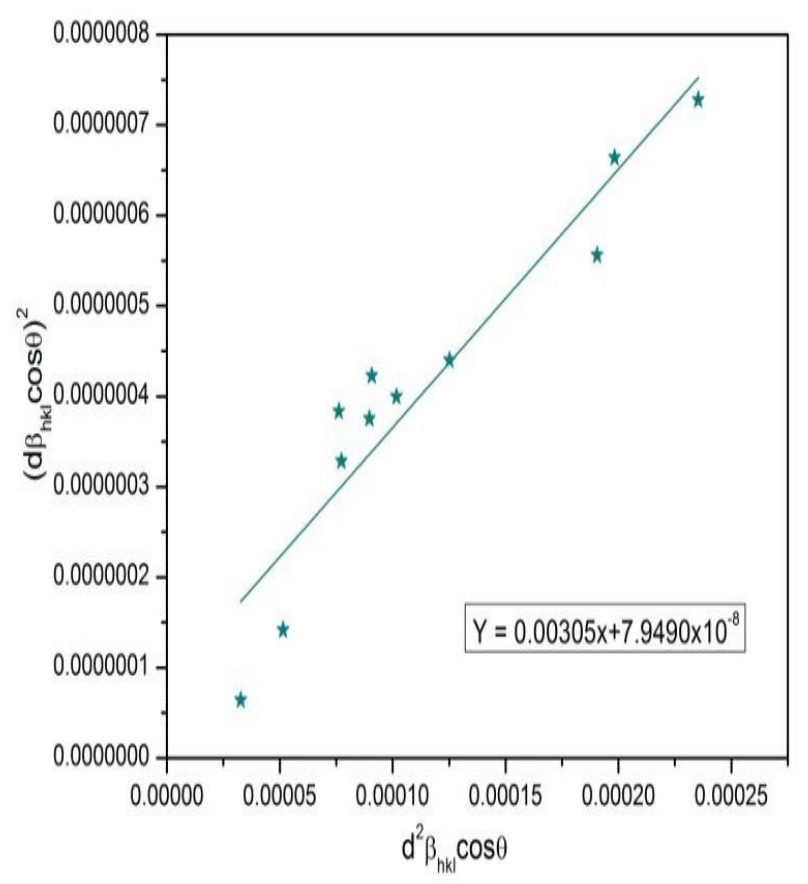

(b)

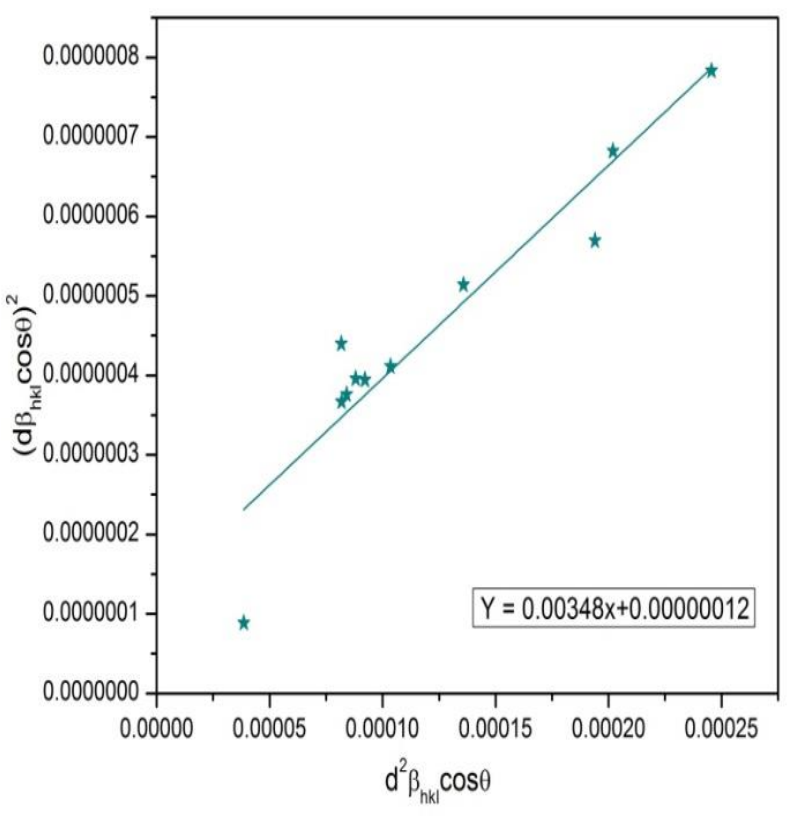

(d) 
Fig. 6 The SSP of undoped and doped ZnO Nano particles. (a) SSP of pure ZnO (b) SSP of 0.50 mol $\% \mathrm{~W}-\mathrm{ZnO}$ (c) SSP of $1.25 \mathrm{~mol} \% \mathrm{~W}-\mathrm{ZnO}$ (d) SSP of $1.75 \mathrm{~mol} \% \mathrm{~W}-\mathrm{ZnO}$.

Table 2. Geometric Analysis of undoped and $\mathrm{W}$ doped $\mathrm{ZnO}$ Nano particles.

\begin{tabular}{|l|c|c|c|r|r|r|r|r|r|r|}
\hline \multicolumn{10}{|c|}{ WILLIAMSONS-HALL ANALYSIS } \\
\hline \multicolumn{10}{|c|}{} & \multicolumn{10}{c|}{$\begin{array}{c}\text { UDM } \\
\text { Samples }\end{array}$} & FWHM & \multicolumn{2}{c|}{ USDM } & \multicolumn{2}{c|}{ UDEDM } & \multicolumn{2}{c|}{ SSP } \\
\hline $\begin{array}{l}\text { Conc. } \\
\text { mol\% }\end{array}$ & & $\begin{array}{c}\mathrm{D} \\
(\mathrm{nm})\end{array}$ & $\begin{array}{c}\epsilon \mathrm{X} \\
10^{-4}\end{array}$ & $\begin{array}{c}\mathrm{D} \\
(\mathrm{nm})\end{array}$ & $\begin{array}{c}\sigma \\
(\mathrm{MPa})\end{array}$ & $\begin{array}{c}\mathrm{D} \\
(\mathrm{nm})\end{array}$ & $\begin{array}{l}\mathrm{U} \\
\left(\mathrm{KJ} / \mathrm{m}^{3}\right)\end{array}$ & $\begin{array}{c}\mathrm{D} \\
(\mathrm{nm})\end{array}$ & $\begin{array}{l}\epsilon \mathrm{X} \\
10^{-4}\end{array}$ \\
\hline Pure & 0.19250 & 44.28 & 1.27 & 44.14 & 8.18 & 44.56 & 24.7 & 44.2 & 3.1 \\
\hline $0.50 \mathrm{~mol} \%$ & 0.20220 & 43.31 & 5.91 & 44.00 & 16.90 & 43.58 & 42.1 & 43.7 & 7.9 \\
\hline $1.25 \mathrm{~mol} \%$ & 0.19636 & 40.64 & 8.33 & 41.37 & 23.60 & 42.38 & 55.0 & 40.1 & 12.5 \\
\hline $1.75 \mathrm{~mol} \%$ & 0.20384 & 39.37 & 9.29 & 39.82 & 24.40 & 40.29 & 61.0 & 38.3 & 12.8 \\
\hline
\end{tabular}

\subsection{Dislocation Density and Crystallinity}

Dislocation density $(\delta)$ in the crystal lattice and crystallinity are calculated using equation (17) and (18) respectively; their values are mentioned in the table 3 corresponding to all the nano-crystals.

$$
\delta=\frac{1}{D^{2}}
$$

$$
\text { Crystallin e\% }=\frac{\text { area of crystallin e peaks }}{\text { area of all peaks }(\text { crstalline }+ \text { amorphous })} \times 100
$$


Table. 3 Morphological Analysis of undoped and W doped ZnO Nano particles.

\begin{tabular}{|c|c|c|c|c|}
\hline Samples & SEM & $\begin{array}{c}\text { Dislocation } \\
\text { Density }\end{array}$ & $\begin{array}{c}\text { Specific Surface } \\
\text { Area }\end{array}$ & \% Crystallinity \\
\hline $\begin{array}{c}\text { Conc. } \\
\text { mol\% }\end{array}$ & $\begin{array}{c}\text { Grain } \\
\text { size(nm) }\end{array}$ & $\left(\mathbf{n m}^{2}\right)$ & $(\mathbf{m} 2 / \mathrm{g})$ & (\%age) \\
\hline $\mathbf{0}$ & $\mathbf{5 5 . 2 8}$ & $\mathbf{0 . 0 0 0 2 2 7 4 9 6}$ & 13.73 & $\mathbf{8 0 . 8 3}$ \\
\hline $\mathbf{0 . 5 0}$ & $\mathbf{5 4 . 7 8}$ & $\mathbf{0 . 0 0 0 2 4 8 0 7 9}$ & $\mathbf{1 4 . 1 2}$ & $\mathbf{8 5 . 3 9}$ \\
\hline 1.25 & $\mathbf{5 2 . 2 8}$ & $\mathbf{0 . 0 0 0 2 5 2 9 9 6}$ & $\mathbf{1 5 . 1 2}$ & $\mathbf{8 7 . 5 5}$ \\
\hline 1.75 & $\mathbf{4 0 . 3 0}$ & $\mathbf{0 . 0 0 0 4 0 1 1 2 2}$ & $\mathbf{1 9 . 8 3}$ & $\mathbf{9 7 . 5 2}$ \\
\hline
\end{tabular}

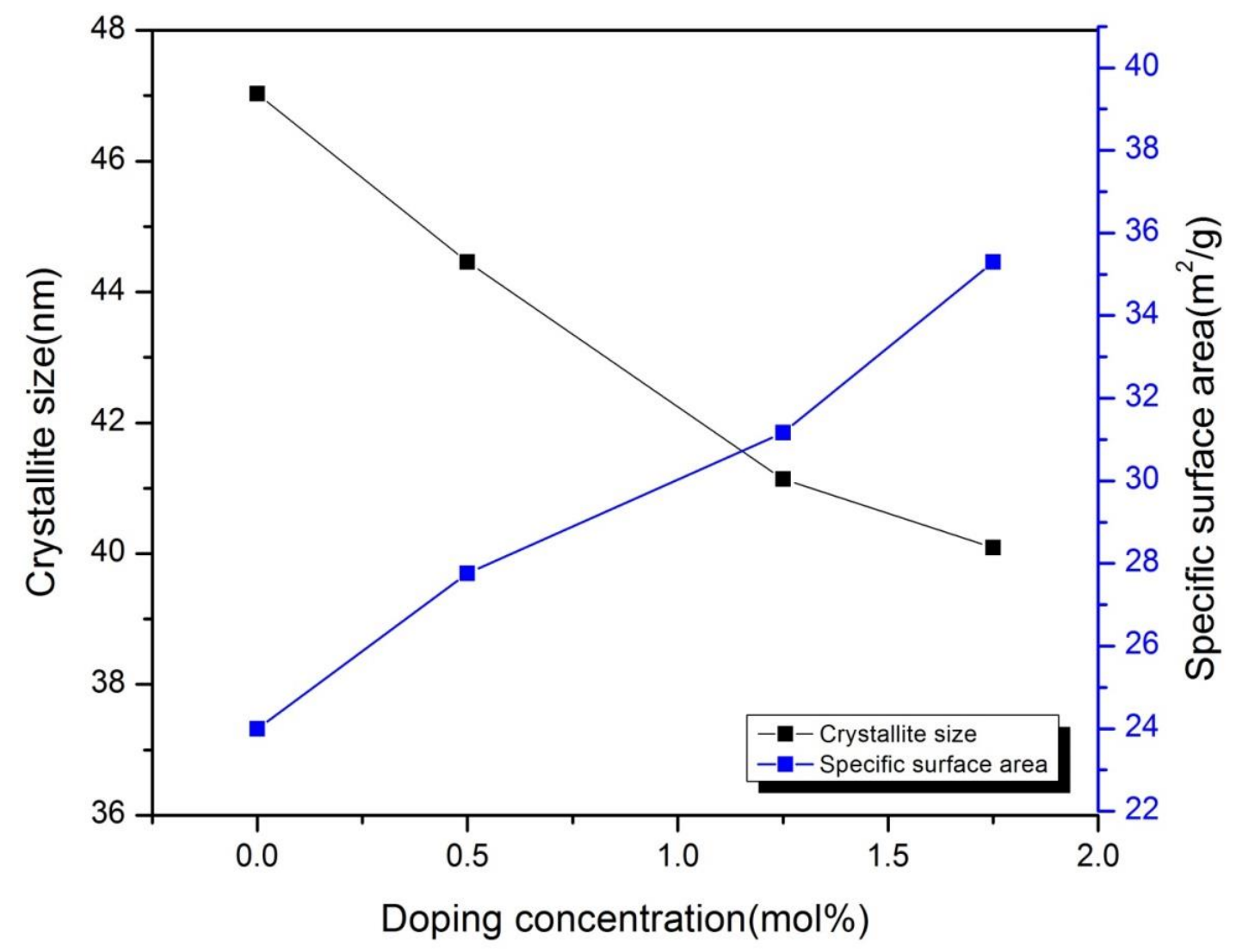

Fig.7 Curve depicting variation of crystallite size and specific surface area with doping concentration. 


\section{SEM Analysis}

Fig. 8 is the inset of the cross-sectional view of the samples in nano dimensional scale. The morphological variations of the samples as observed by scanning electron microscope reveals nanostructured self-assembly of hexagonal columnar growth with smooth edges present viably. Prepared zinc oxide nanostructures may be characterized by connected sinter-necked grains with sufficient porosity offering large specific area and enough available sites for water molecule percolation providing effective humidity response. The statistical analysis of the micrograph indicates a uniform distribution of grains of diameter from $55.28 \mathrm{~nm}$ to $40.60 \mathrm{~nm}$. A decrement in grain size is observed as the doping progresses from $\mathrm{x}=0$ to $1.75 \mathrm{~mol} \%$, this variation in the doping concentration affects the $\mathrm{ZnO}$ crystal growth morphology and formation of aggregates. In this self-assembly the nanostructures are distributed evenly throughout the crystal. At a doping percentage of $1.75 \mathrm{~mol} \%$ there is an appearance of one-dimensional ricelike nanostructure and at some sites the nanostructures appear to have embedded into one another. For an increased concentration of W6+ ions there is an increased availability of nucleation sites resulting in polycrystalline aggregates and sufficient growth of nanostructures. The Specific Surface Area (SSA) is determined from equation (19);

$$
S=\frac{6}{\rho \times D_{m}}
$$

Where, $\rho$ is the bulk density of $\mathrm{ZnO}$ and $\mathrm{Dm}$ is the average particle size and 6 is the shape factor [34]. The SSA for the prepared $\mathrm{ZnO}$ nano-particles is mentioned in Table.3. 


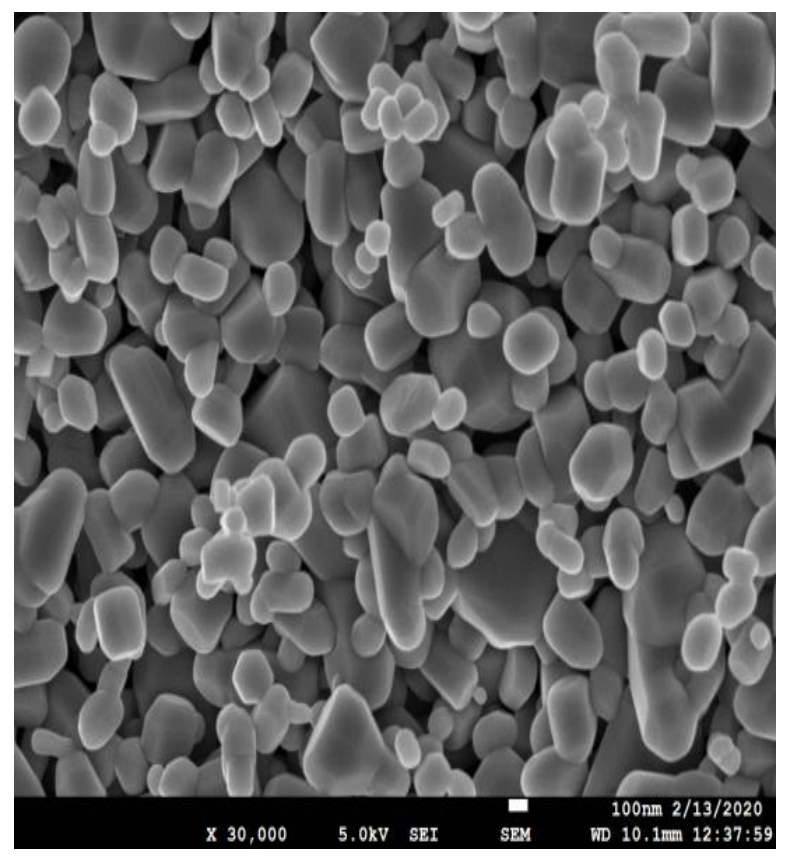

(a)

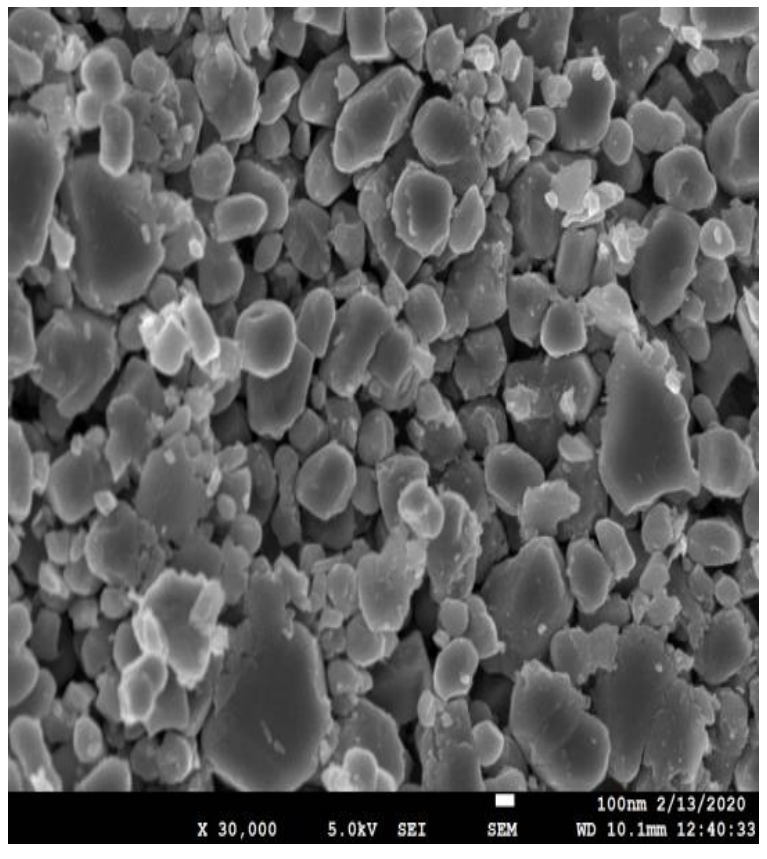

(b)

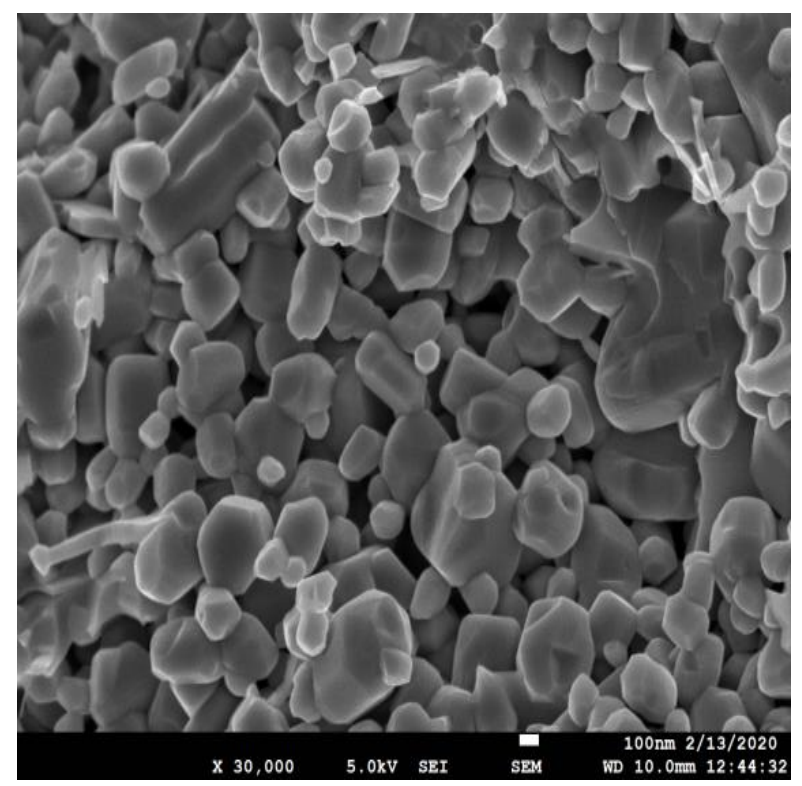

(c)

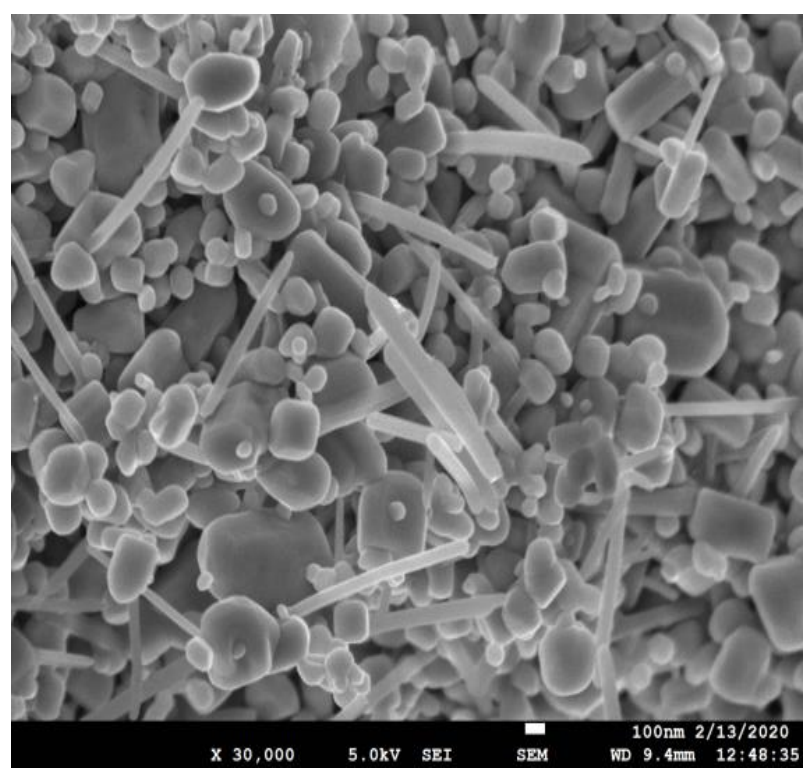

(d) 
Fig.8 SEM micrographs of undoped and doped ZnO Nano particles. (a) Pure ZnO (b) 0.50 mol\% W-ZnO (c) 1.25 mol\% W-ZnO (d) 1.75 mol\% W-ZnO

\section{Humidity Sensing}

A typical humidity response curve for the pure and $\mathrm{W}$ doped $\mathrm{ZnO}$ sensors in humidity range of $10 \%$ to $90 \% \mathrm{RH}$, is demonstrated in Fig.9. Relative humidity sensors are popular and can be classified as ceramic, semiconductor, and polymer humidity sensors. Present work deals with the fabrication and investigation of performance of a resistive type, semiconductor metal oxide humidity sensor. As per the humidity sensing response of all the prepared sensors, when the samples are exposed to a humidity range of $10 \%$ to $90 \% \mathrm{RH}$, their resistance shows a decrement. The $\mathrm{ZnO}$ samples show quick and steady response for sufficient range of resistance variation vs. relative humidity.

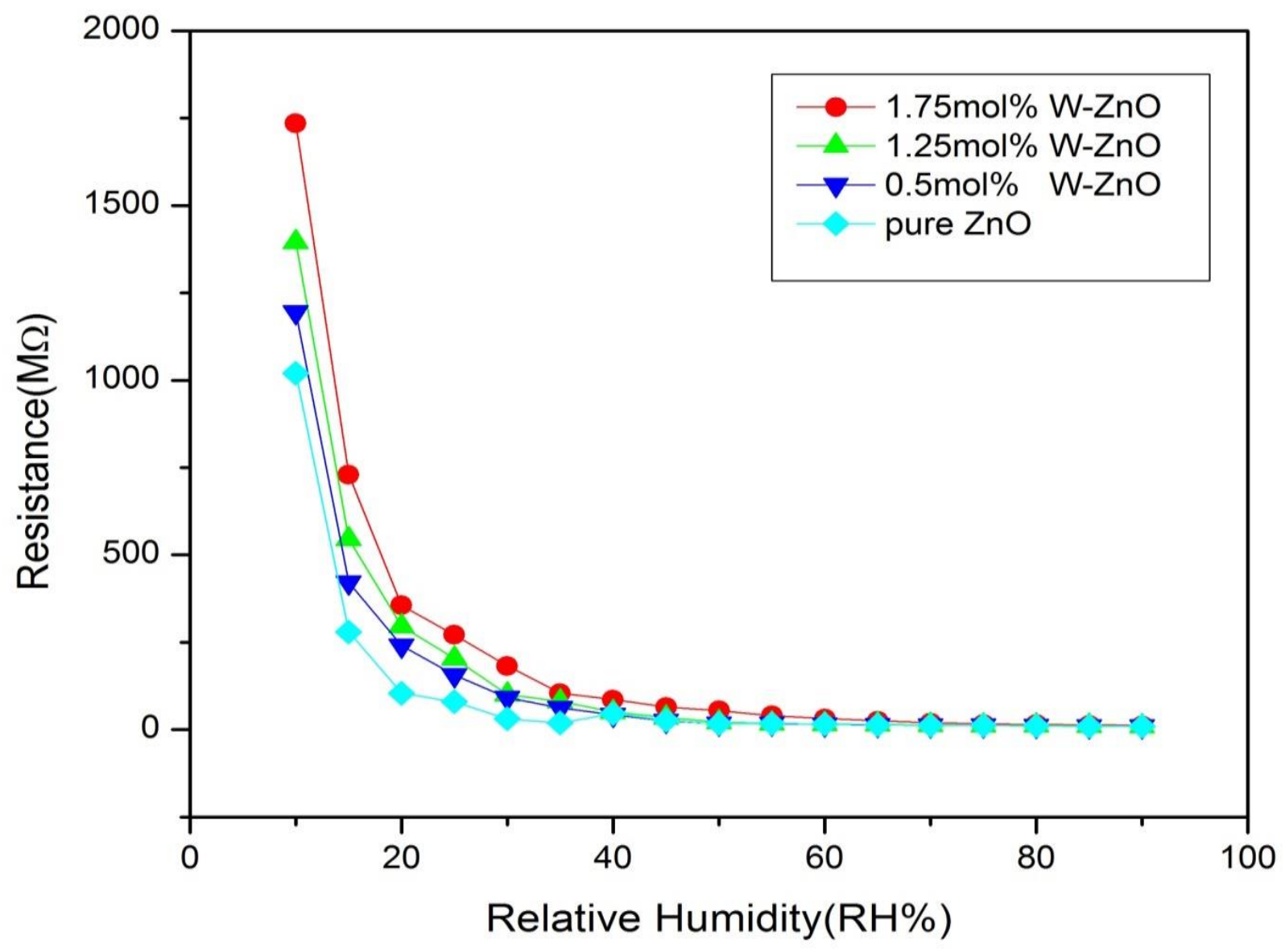


Fig.9 Relative humidity curve for pure and $\mathrm{W}$ doped $\mathrm{ZnO}$ nano-sensors.

The variation of resistance as a function of $\mathrm{RH} \%$ is predominantly due adsorption of water molecule to the metal oxide grains over the entire surface of the sensor. Water molecule being of almost same pores of the sensor samples readily gets adsorbed on the surface, leading to saturation in porous metal-oxide succeeded by capillary condensation through nanopores for higher ambient moisture $[35,36]$. The capillary condensation builds up an aqueous medium responsible for electrolytic conduction [37].

Abundance of $\mathrm{Zn}^{2+}$ ions at the surface provides a high charge density and strong electrostatic field to facilitate chemisorption of water molecule. For relatively lower RH\% the water molecule gets chemisorbed to the metal oxide porous surface and self-ionizes into $\mathrm{OH}$ - hydroxyl ion and $\mathrm{H}+$ ion [38].

$$
\mathrm{H}_{2} \mathrm{O} \Leftrightarrow \mathrm{H}^{+}+\mathrm{OH}^{-}
$$

The hydroxyl ion $\left(\mathrm{OH}^{-}\right)$forms a covalent bond with the cation of the nano-sensor i.e. $\mathrm{Zn}^{2+}$ and releasing an $\mathrm{H}^{+}$ion (proton). Therefore, at lower $\mathrm{RH} \%$ the conduction occurs through chemisorbed layer by proton hopping mechanism [39]. As the humidity further increases a layer of water molecules is physiosorbed onto the previously chemisorbed layer of hydroxyl ions by active interaction of water molecules with the $\mathrm{Zn}^{2+}$ surface. The physiosorbed water layer facilitates formation of hydronium ion $\mathrm{H}_{3} \mathrm{O}$.

$$
\mathrm{H}_{2} \mathrm{O}+\mathrm{H}_{2} \mathrm{O} \Leftrightarrow \mathrm{H}_{3} \mathrm{O}^{+}+\mathrm{OH}^{-}
$$


At even higher humidity levels a continuum multilayer of water is formed, followed by dissociation of water molecule leading to the formation of mobile proton and the charge conduction takes place through proton transfer between adjacent sites by Grotthuss chain reaction [40], which causes a decrease in resistance due to increase in charge carriers for higher water content at high humidity values. This reduction in resistance with increasing humidity values is almost exponential.

The micro-processes involved during the humidification accounts for the variation of resistance with humidity variance. For relatively lower values of ambient moisture a monolayer of water molecules is chemisorbed on the nano-sensor surface by self- ionization forming covalent bond with the surface cation.

The presence of sufficient pores due to addition of tungsten promotes the adsorption process on the nano-porous surface enhancing the humidity response. The decrease in crystallite size suggest large specific surface area which is desired for better moisture adsorption. This is also evident from the electrical response of the nano sensors with respect to humidity. The nano sensor having smaller crystallite size possesses large specific surface and better humidity response.

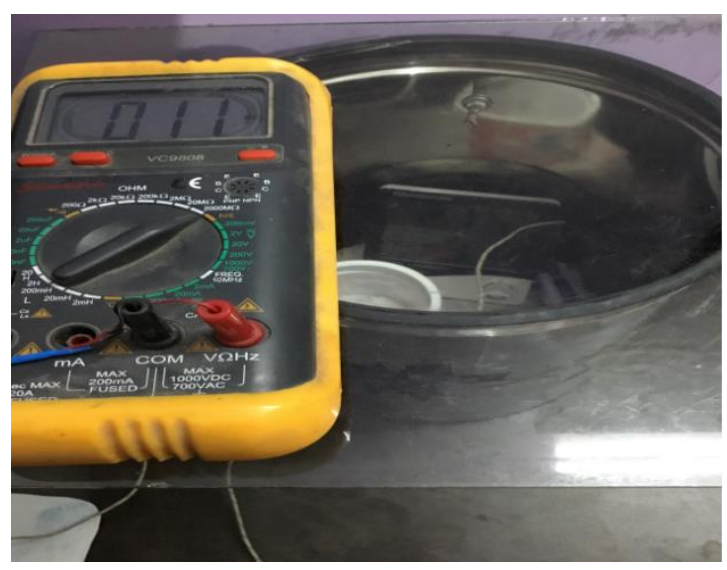

Fig. 10 The experimental setup for Relative Humidity Sensing measurement. 


\subsection{Response and recovery time}

The two factors determining the operational efficiency of the device are response and recovery time. Response/recovery time is the stimuli to the adsorption and desorption of water molecule at the surface of the nano sensor. The adsorption and desorption occur via different mechanisms. The water vapors molecule bonded with the surface of the sensor requires external energy to get liberated thereby making it an endothermic process. The breaking of bonds may require a longer duration hence recovery time may be longer than the response time. Adsorption of water molecule releases energy, hence, is and exothermic process. The desorption kinematics is slower than adsorption as dissociation of hydrogen bond between the oxide cation and hydroxyl group requires activation energy.

The response time $\left(\tau_{\mathrm{s}}\right)$ for all the samples is recorded by instantaneously switching the relative humidity from 10 to $90 \mathrm{RH} \%$ and recovery time $\left(\tau_{c}\right)$ is measured by the rate at which the sensor responds to the change in relative humidity (RH\%) by a sudden change of humidity from 90 to $10 \mathrm{RH} \%$ during desiccation. Fig. 11 corresponds to the response/recovery time of $1.75 \mathrm{~mol} \% \mathrm{~W}-$ $\mathrm{ZnO}$, the response and recovery time of the individual nanosensor samples are mentioned in Table.4. As obvious presence of nano-rice structure drastically decreases the response and recovery time of the nano-sensors. 
Table. 4 Response and Recovery Time for pure and doped $\mathrm{ZnO}$ Nano particles

\begin{tabular}{|c|c|c|c|}
\hline \multicolumn{4}{|c|}{ Humidity sensor parameters } \\
\hline Samples & Sensitivity & Response Time & Recovery Time \\
\hline $\begin{array}{l}\text { (Doping } \\
\text { mol\%) }\end{array}$ & & Secs & Secs \\
\hline 0 & 102 & 120 & 180 \\
\hline 0.5 & 120 & 95 & 152 \\
\hline 1.25 & 127 & 75 & 138 \\
\hline 1.75 & 144 & 45 & 105 \\
\hline
\end{tabular}

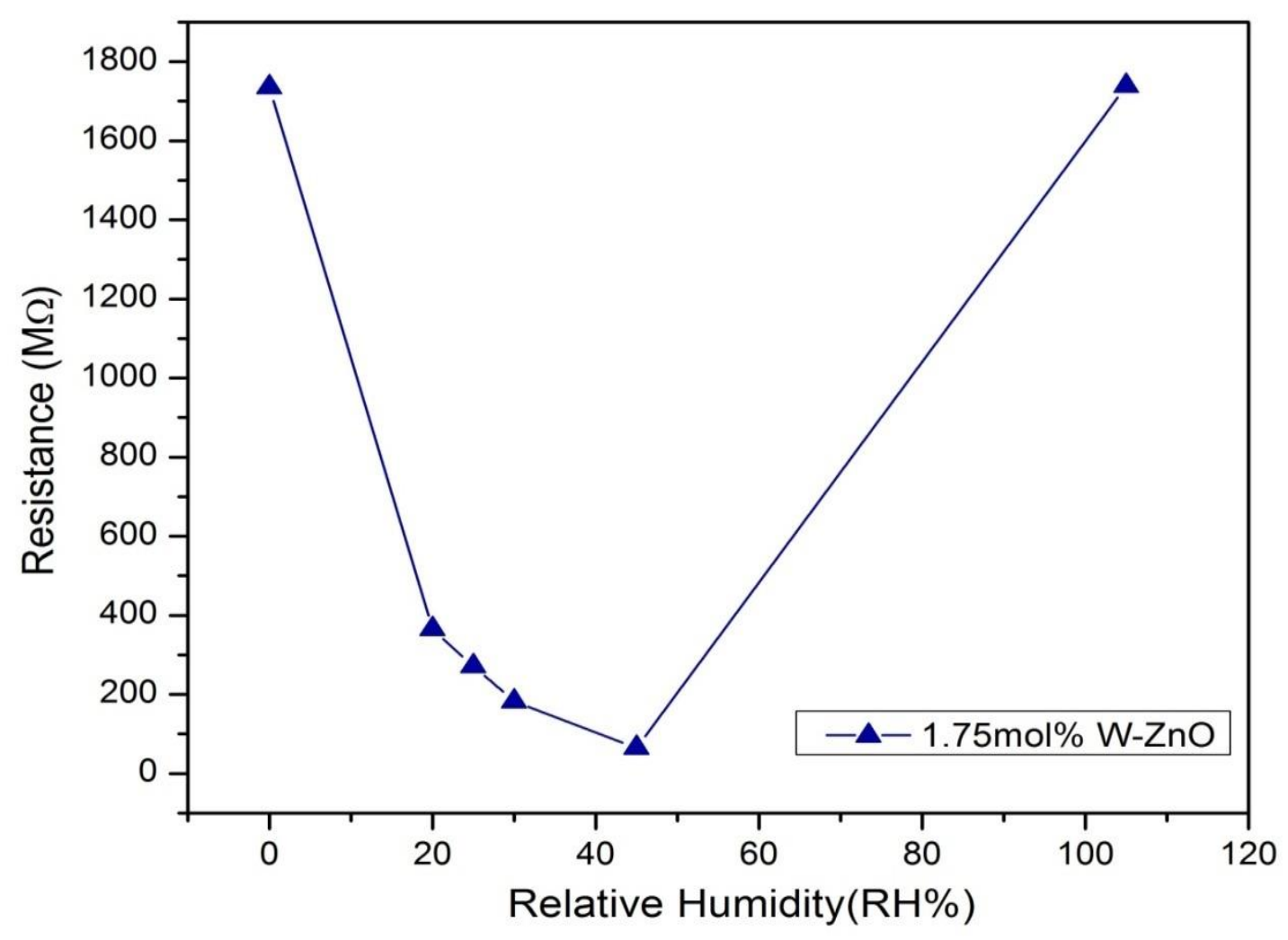

Fig.11 Response and Recovery time graph for $1.75 \mathrm{~mol} \% \mathrm{~W}-\mathrm{ZnO}$ humidity sensor. 


\subsection{Sensitivity}

Sensitivity is a good indicator of the performance of the humidity sensor. The values for sensitivity of $\mathrm{W}^{6+}$ doped $\mathrm{ZnO}$ humidity sensors are deduced from equation (20). The values obtained for each sensor is mentioned in Table.4.

$$
\text { Sensitivity }=\frac{R_{i}}{R_{r h}}
$$

Where, $\mathrm{R}_{\mathrm{i}}$ resistance corresponding to initial value of humidity, $\mathrm{R}_{\mathrm{rh}}$ is sample resistance for different $\mathrm{RH} \%$ [41]. The sensitivity values for each $\mathrm{W}^{6+}$ doped $\mathrm{ZnO}$ nano-sensors exhibit high sensitivity values and is highly responsive to $\mathrm{RH} \%$ and the amount of $\mathrm{W}^{6+}$ present in the nanosensors. Hence incorporation of tungsten is effective in increasing the sensitivity of $\mathrm{ZnO}$ based humidity sensors. $\mathrm{ZnO}_{1-\mathrm{x}} \mathrm{W}_{\mathrm{x}}$ for $\mathrm{x}=1.75 \mathrm{~mol} \%$ shows higher sensitivity values up to $144 \mathrm{x}$ observed at $90 \mathrm{RH} \%$ among other samples. Improved sensitivity in these nano-sensors may be attribute to decreased crystallite size because of tungsten incorporation, the reduced grain size offers higher crystallinity (supported by data in Table.3) [42] and larger active surface for moisture adsorption. The high sensitivity can also be credited to the nano-rice structure formation of grain size of $15 \mathrm{~nm}$ which may have intensified the humidity mechanism by providing abundant grain boundaries and nanopores for capillary action. 


\subsection{Stability and Aging effects}

Long term stability ensures high efficiency of the sensors. Fig.12 are the insets of stability curves for $\mathrm{ZnO}_{1-\mathrm{x}} \mathrm{W}_{\mathrm{x}}$ for $\mathrm{x}=1.75 \mathrm{~mol} \%$ sensor deduced by measuring impedances $<90 \%$ RH for at least 270 days which show slight deviation of resistances for each cycle over the period. The aging effects are supposed to degrade the working of the sensors owing to extended exposure of sensor surface to humidity, loss of surface cations due to thermal diffusion, inhibition of cationic sites from adsorbed contaminants making the surface less reactive.

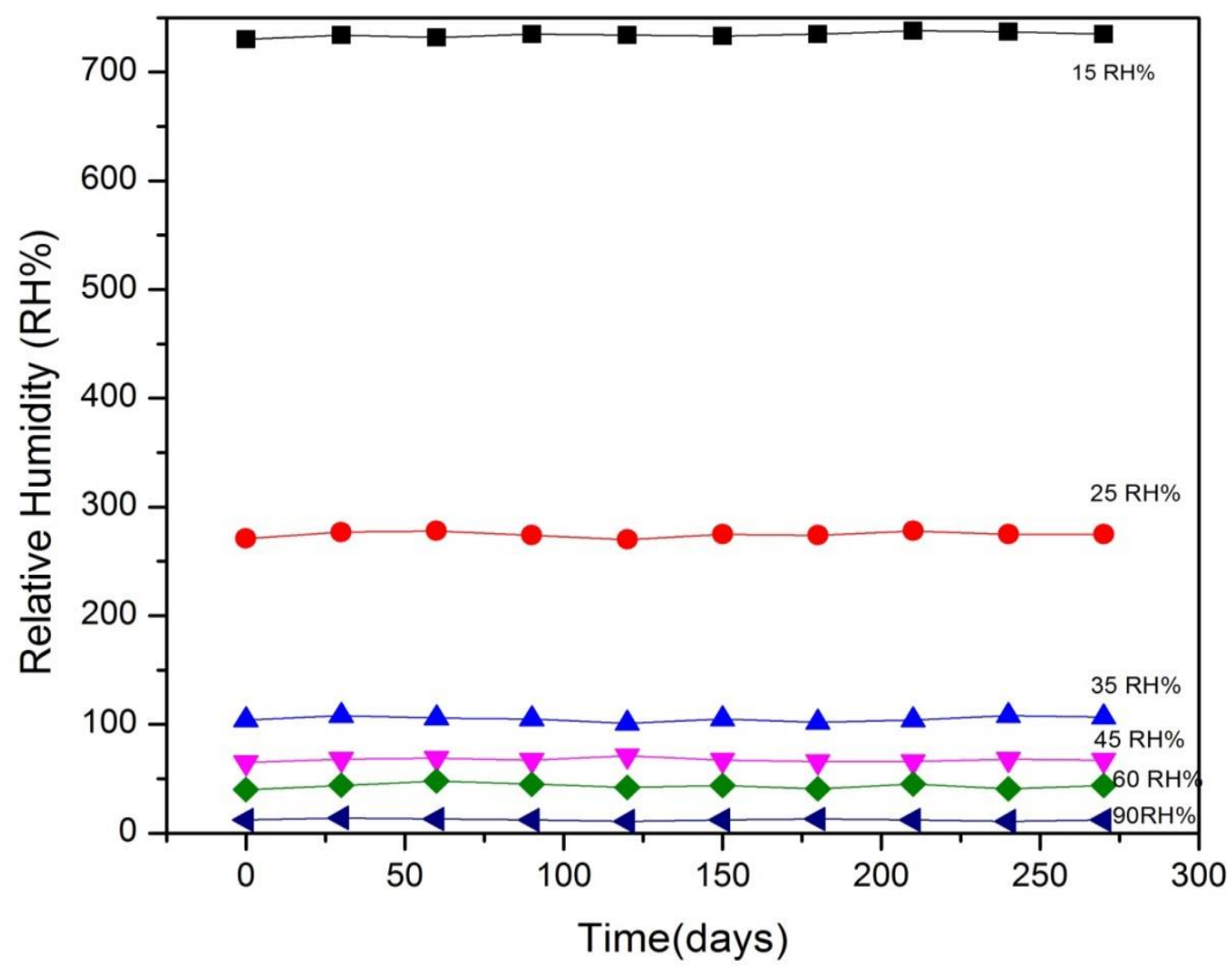

Fig.12 Stability Curve for $1.75 \mathrm{~mol} \% \mathrm{ZnO}$ sensor 


\subsection{Hysteresis}

A certain amount of deviation in adsorption curve during humidification and desorption curve during desiccation is observed which gives rise to hysteresis. The processes of desorption of moisture which is chemisorbed on metal oxide surface requires greater energy in contrast to adsorption. Hence desorption of water molecule requires additional energy there by leading to hysteresis during the dehumidification process. Hysteresis is a prominent factor in determining the reliability of the humidity sensor, a credible sensor is expected have minimum hysteresis error $\left(\gamma_{\mathrm{H}}\right)$ which is calculated at different $\mathrm{RH} \%$ values for all $\mathrm{W}-\mathrm{ZnO}$ based sensors from equation (21).

$$
\gamma_{H}= \pm \frac{\Delta R_{\max }}{2 F_{f s}}
$$

Here $\gamma_{H}$ is the hysteresis error as a ratio of maximum difference in output during humidification and desiccation $\left(\Delta R_{\max }\right)$ to full scale output $\left(\mathrm{F}_{\mathrm{fs}}\right)$ [43]. Fig.13 depicts the hysteresis curve for $\mathrm{ZnO}_{1-\mathrm{x}} \mathrm{W}_{\mathrm{x}}$ for $\mathrm{x}=1.75 \mathrm{~mol} \%$ sensor. The hysteresis error is negligible for all the samples making them more reliable for practical usage. The minimum hysteresis error detected is 8.24 for $\mathrm{ZnO}_{1 \text { - }}$ ${ }_{\mathrm{x}} \mathrm{W}_{\mathrm{x}}$ for $\mathrm{x}=1.75 \mathrm{~mol} \%$ sensor while other sensors have $11.0,12.38$ and 12.72 error rates for $\mathrm{x}=$ $1.25,0.50$ and pure $\mathrm{ZnO}$ respectively. 


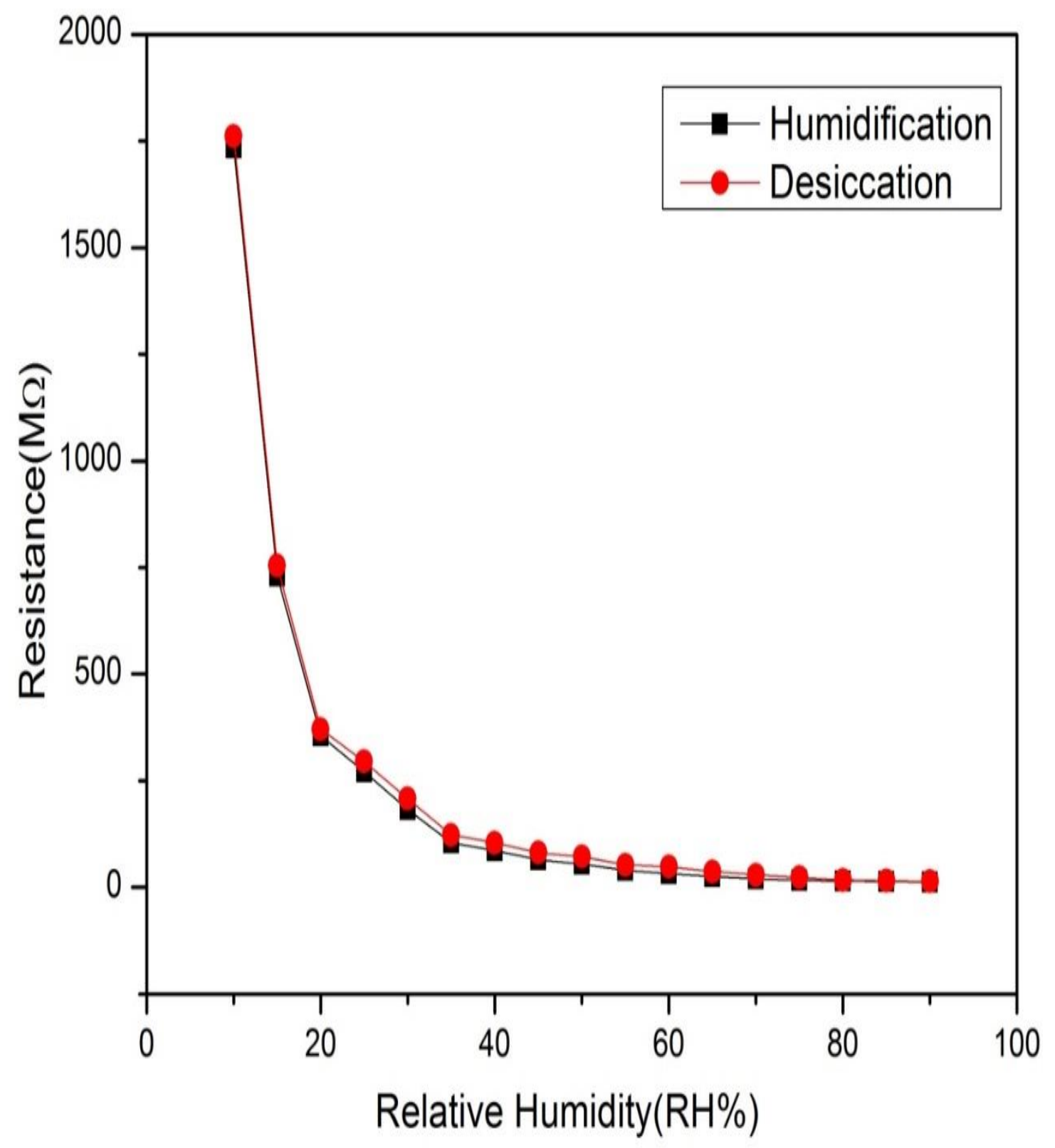

Fig. 13 Hysteresis curve for $1.75 \mathrm{~mol} \% \mathrm{~W}$ doped $\mathrm{ZnO}$ nano-sensor. 


\section{Conclusion}

Nano-scaled tungsten doped $\mathrm{ZnO}$ exhibiting different morphologies are prepared using co-precipitation synthesis route for structural and humidity sensing analysis. X-ray diffractogram reveals that the nanoparticles possess hexagonal wurtzite structure. Systematic variation of tungsten concentration has led to decrease in crystallite size of the nano-crystalline $\mathrm{ZnO}$. Scherrer's formula, modified versions of W-H analysis and size-strain plot procedure studies the line broadening of the nanoparticles induced from small crystallite size and lattice-strain. The values crystallite size calculated from Scherrer's formula and W-H analysis are in good agreement with each other. SEM analysis affirms of hexagonal columnar structure of the synthesized nano-structures and formation of nano-rice for $1.75 \mathrm{~mol} \%$ of $\mathrm{W}-\mathrm{ZnO}$. The humidity sensing characteristics are particularly dependent on the $\mathrm{W}$ concentration in ZnO. Nano-sensor doped with $1.75 \mathrm{~mol} \%$ of tungsten has highest sensitivity of 144x in 10-90\%RH range. The response and recovery time for $1.75 \mathrm{~mol} \% \mathrm{~W}-\mathrm{ZnO}$ is recorded to be $45 \mathrm{sec}$ and $105 \mathrm{sec}$ respectively which are faster to the humidity sensors mentioned in the literature. The sensors show hysteresis within acceptable range which makes them efficient for practical usage. All the $\mathrm{W}-\mathrm{ZnO}$ sensors are stable for resistance measurements over a period of 270 days. This sensor exhibits lower values of hysteresis, aging effects, shows higher stability for over a period of 9 months. All these factors make these nano humidity sensors credible for their use in practical environment. 


\section{Acknowledgments}

Authors owe their gratitude to the Department of Physics, University of Lucknow for providing X-ray diffraction facility for characterization.

\section{References}

[1] Z M. Rittersma*,Recent achievements in miniaturized humidity sensors-a review of transduction techniques, Sensors and Actuators A 96 (2002), 196-210.

[2] Z.YuanH .Tai, X.Bao ,C.Liu ,Z.Ye, Y.Jiang ,Enhanced humidity-sensing properties of novel graphene oxide/zincoxidenano particles layered thin film QCM sensor, Mater.Lett.174(2016)28-31.

[3] Vandana Shakya*, N K Pandey, Suneet Kumar Misra and Akash Roy, Electrical and optical properties of $\mathrm{ZnO}-\mathrm{WO}_{3}$ nanocomposite and its application as a solid-state humidity sensor, Bull. Mater. Sci., Vol. 40, No. 2, April 2017, pp. 253-262. Indian Academy of Sciences ,DOI: 10.1007/s12034-017-1373-5.

[4] A. Khorsand Zak, W.H. Abd. Majid , M. Ebrahimizadeh Abrishami , Ramin Yousefi , R. Parvizi , Synthesis, magnetic properties and X-ray analysis of Zn0.97X0.03O nanoparticles (X 1/4 Mn, Ni, and Co) using Scherrer and size-strain plot methods, doi:10.1016/j.solidstatesciences.2012.01.019. 
[5] R.R. POTTER, Enhanced Photocurrent $\mathrm{ZnO} / \mathrm{CdS} / \mathrm{CuInSe} 2$ Solar cells, https://doi.org/10.1016/0379-6787(86)90107-9.

[6] Karima Bouras*a, Guy Schmerber ${ }^{\mathrm{d}}$, Damien Aureau ${ }^{\mathrm{b}}$, Hervé Rinnert ${ }^{\mathrm{c}}$,Jean-Luc Rehspringer $^{\mathrm{d}}$,Dris Ihiawakrim $^{\mathrm{d}}$,Aziz Dinia $^{\mathrm{d}}$,Abdelilah Slaouia and Silviu Colis, Photon management properties of $\mathrm{Yb}$-doped $\mathrm{SnO}_{2}$ nanoparticles synthesized by the sol-gel technique, https://doi.org/10.1039/C9CP01993F.

[7] R. Elilarassi , G.chandrasekaran, Synthesis, structural and optical characterization of Nidoped $\mathrm{ZnO}$ nanoparticles, Journal of Materials Science: Materials in Electronics 22, pages751756(2011).

[8] R.SwapnaaT.SrinivasaReddyaK.Venkateswarlub M.C. Santhosh Kumara, Effect of PostAnnealing on the Properties of $\mathrm{Eu}$ Doped $\mathrm{ZnO}$ Nano Thin Films. https://doi.org/10.1016/j.mspro.2015.06.085.

[9] Y. R. Shinde, T. P. Gujar, C. D. Lokhande, R. S. Nane and S. H. Han, Bismuth oxide thin films by chemical bath deposition method: Annealing effect, Materials Chemistry and Physics, Vol. 326,2006.Doi: 10.1016/j.apsusc.2004.12.050. 
[10] Shufeng Si , Shuo Li, Zhengqiu Ming, Linpei Jin, Humidity sensors based on ZnO Colloidal nanocrystal clusters, Chemical Physics Letters, pp.288-291, 2010,

Doi: 10.1016/j.cplett.2010.05.013.

[11] M. H. Koch, P. Y. Timbrell and R. N. Lamb, The Influence of Film Crystallinity on the Coupling Efficiency of $\mathrm{ZnO}$ Optical Modulator Waveguides, Semiconductor Science and Technology, Vol. 10, No. 11, 1995, p. 1523.

[12]Roya Ebrahimi, Afshin Maleki, Yahya Zandsalimi, Reza Ghanbari, Photocatalytic degradation of organic dyes using WO3-doped $\mathrm{ZnO}$ Nanoparticles fixed on a glass surface in aqueous solution, Journal of Industrial and Engineering Chemistry ( IF 5.278 ), DOI: 10.1016/j.jiec.2019.01.041.

[13] W. Shana , W. Walukiewicz, J. W. Ager III, and K. M. Yu, Nature of room-temperature $\begin{array}{lllll}\text { photoluminescence } & \text { in } \quad \text { ZnO, Appl. } & \text { Phys. } & \text { Lett. 86, } & 1911\end{array}$ (2005), https://doi.org/10.1063/1.1923757.

[14] Priya Gupta, Savita Maurya, Narendra Kumar Pandey and Vernica Verma, Metal-oxide Based Ammonia Gas Sensors - A Review, Nanoscience \&amp; Nanotechnology-Asia (2020) 10: 1. https://doi.org/10.2174/2210681210999200718005402 
[15] S. Misra, N. K. Pandey, V. Shakya, Activation energy and humidity / gas sensing studies of $\mathrm{Al}$ doped $\mathrm{ZnO}$ nanomaterials; Corpus ID: 14112825.

[16] Francisco Pola-Albores, ${ }^{1}$ Francisco Paraguay-Delgado, ${ }^{1}$ Wilber Antúnez-Flores, ${ }^{1}$ Patricia Amézaga-Madrid, ${ }^{1}$ Edna Ríos-Valdovinos, ${ }^{1}$ and Mario Miki-Yoshida, Microstructural Study of ZnO Nanostructures by Rietveld Analysis, Volume 2011|Article ID 643126 | https://doi.org/10.1155/2011/643126.

[17] Effect of firing temperature on humidity sensing properties of $\mathrm{SnO} 2$ thick film resistor, R.Y Borse and A.S. Grade, sensors \& transducers Journal Vol. 111, Issue 12, December 2009,pp. 155-165.

[18] M. F Malek ${ }^{1,2,3}$, M.M. Yusoff ${ }^{1}$, N.D. Md. Sin $^{1}$, A.S. Zoolfakar ${ }^{1}$, Z. Khusaimi ${ }^{2,3}$, M. Rusop $^{1,2}$,S.A. Saidi ${ }^{1}$, M..H. Mamat ${ }^{1,2}$, A.S. Ismail, Humidity sensing properties of Al-doped zinc oxide coating films. doi: 10.1063/1.5036863.

[19] Gupta, P., Maurya, S., Pandey, N.K., Vernica, V., Structural, electrical and humidity Sensing properties of nano-structured nickel oxide prepared by sol-gel method. J Mater Sci: Mater Electron (2021). https://doi.org/10.1007/s10854-020-05099-4. 
[20] Zhengwu Jin, T. Fukumura, and M. Kawasaki, High throughput fabrication of transitionmetal-doped epitaxial $\mathrm{ZnO}$ thin films: A series of oxide-diluted magnetic semiconductors and their properties, Appl. Phys. Lett. 78, 3824 (2001). https://doi.org/10.1063/1.1377856.

[21] A Simimol ${ }^{1,2}$, Aji A Anappara ${ }^{2}$ and Harish C Barshiliaa ${ }^{1}$, Influence of defects on electrical properties of electrodeposited co-doped ZnO nanocoatings, DOI: 10.1088/2053-1591/aa5135.

[22] Suhasini Kunchakara, ,Meenakshi Dutt, Amar Ratan, Jyoti Shah, Vaishali Singh, R. K. Kotnala, Synthesis and characterizations of highly ordered $\mathrm{KCl}-\mathrm{MCM}-41$ porous nanocomposites for impedimetric humidity sensing, DOI: 10.1007/s10934-018-0613-4

[23] Milind Bodke ${ }^{1, *}$, Umesh Gawai $^{2}$, Ashok Patil ${ }^{2}$, and Babasaheb Dole ${ }^{3}$, Estimation of accurate size, lattice strain using Williamson-Hall models, SSP and TEM of Al doped $\mathrm{ZnO}$ nanocrystals, https://doi.org/10.1051/mattech/2018055

[24] Ü. Özgüra, Ya. I. Alivov, C. Liu, A. Tekeb, M. A. Reshchikov, S. Doğanc, V. Avrutin, S.-J. Cho, and $\mathrm{H}$. Morkoçd, A comprehensive review of $\mathrm{ZnO}$ materials and devices, https://doi.org/10.1063/1.1992666. 
[25] Yichuan Chen $1^{1}$, Yuehui $\mathrm{Hu}^{1}$, Xiaohua Zhang ${ }^{1}$, Keyan $\mathrm{Hu}^{1}$,Fan Tong ${ }^{1}$. Zixuan $\mathrm{Lao}^{1}$, Weiqiang Shuai ${ }^{1}$, Investigation of the properties of $\mathrm{W}$-doped $\mathrm{ZnO}$ thin films with modulation power deposition by RF magnetron sputtering, DOI: 10.1007/s10854-016-6211-9.

[26] Ramin Yousefia, FaridJamali, Sheinib, Abdolhossain Sa'aedic A. Khorsand Zakd Mohsen Cheraghizadec Siamak Pilban Jahromie Nay Ming Huange, Influence of lead concentration on morphology and optical properties of $\mathrm{Pb}$-doped $\mathrm{ZnO}$ nanowires, DOI: 10.1016/j.ceramint.2013.05.008

[27] Wang, Z.L. Zinc oxide nanostructures, properties and applications, J.Phys. Condens. Matter 2004,16,829-858.

[28] A.Khorsand Zak, W.H. Abd.Majid, M.E. Abrisham, Ramin Yousfi, X-ray of ZnO nanoparticles by Williamson-Hall and size-strain plot methods, Solid State Sciences 13(2011)251-256.

[29] Yendrapati Taraka Prabhu ${ }^{1 *}$, Kalagadda Venkateswara Rao ${ }^{1}$, Vemula Sesha Sai Kumar ${ }^{1}$, Bandla Siva Kumari ${ }^{2}$, X-Ray Analysis by Williamson-Hall and Size-Strain Plot Methods of ZnO Nanoparticles with Fuel Variation, http://dx.doi.org/10.4236/wjnse.2014.41004.

[30] Kumar, R Hymavathi, X-ray peak profile analysis of solid-state sintered alumina doped zincoxide ceramics by Williamson- Hall and size-strain plot methods, JASCER, 260 (2017). 
[31] J.F. Nye, Physical Properties of Crystals: Their Representation by Tensors and Matrices, Oxford, New York, 1985.

[32] M. Augustin, T. Balu, Estimation of Lattice Stress and Strain in Zinc and Manganese Ferrite Nanoparticles by Williamson-Hall and Size-Strain Plot Methods, DOI: 10.1142/S0219581X16500356.

[33] M.A. Tagliente, M.Massaro, Strain-driven $\left(\begin{array}{lll}0 & 0 & 2\end{array}\right)$ preferred orientation of $\mathrm{ZnO}$ nanoparticles in ion-implanted silicall Nucl. Instrum Methods Phys. Res. B. Vol. 266, 2008, pp.1055-1061.

[34] B.C. Yadav*, Saroj Radheshyam Sabhajeet, Rakesh Kumar Sonker, Sol-gel formed grape-like nanostructured titania based liquefied petroleum gas sensor, DOI: 10.29011/JMSR-108/100008.

[35] Qin Kuang, Changshi Lao, Zhong Lin Wang, Zhaoxiong Xie, and Lansun Zheng; High sensitivity humidity sensor based on a single $\mathrm{SnO}_{2}$ nanowire,https://doi.org/10.1021/ja070788m.

[36] J.H. Anderson and G.A. Parks, The electrical conductivity of silica gel in the presence of adsorbed water, Z Phys. Chem., 72 (1968) 3362-3368 
[37]S.S. Pingale ${ }^{\text {a }}$, S.F. Patil ${ }^{\text {a }}$, M.P. Vinod ${ }^{b}$, G. Pathak ${ }^{b}$, K. Vijayamohanan, Mechanism of humidity sensing of Ti-doped $\mathrm{MgCr}_{2} \mathrm{O}_{3}$, Materials Chemistry and Physics 46 ( 1996) 72-76.

[38]Akshay Kumara, Babankumar S. Bansod ${ }^{\text {b }}$, Tejbir Singh ${ }^{\mathrm{c}}$, Anup Thakurd, Tarandip Singhe and Jeewan Sharma ${ }^{*}$, Harinder Singha, Enhanced moisture sensing properties of a nanostructured $\mathrm{ZnO}$ coated capacitive sensor, DOI: 10.1039/C7RA10917B.

[39] B.C. Yadav, Richa Srivastava \&C.D. Dwivedi, Synthesis and characterization of ZnO-TiO2 nanocomposite and its application as a humidity sensor, https://doi.org/10.1080/14786430802064642.

[40] Hamid Farahani, Rahman Wagiran and Mohd Nizar Hamidon; Humidity sensors Principle, mechanism, and fabrication technologies: A Comprehensive review.

Doi: 10.3390/s140507881.

[41] Gokhan Algun, Namik Akcay, Enhanced sensing characteristics of relative humidity sensors based on $\mathrm{Al}$ and $\mathrm{F}$ co-doped $\mathrm{ZnO}$ nanostructures thin films; Journal of Material Science: Materials in Electronics, https://doi.org/10.1007/s10854-019-01982-x. 
[42] Weon-Pil Taia Jun-Gyu Kima Jae-Hee Ohb, Humidity sensitive properties of nanostructured Al-doped $\mathrm{ZnO}: \mathrm{TiO}_{2}$ thin films, https://doi.org/10.1016/S0925-4005(03)00602-6.

[43] Yogendra Kumar, Alfa Sharma and Parasharam M. Shirage, Shape-controlled CoFe2O4 nanoparticles as an excellent material for humidity sensing, DOI:10.1039/c7ra11072c. 
Figures

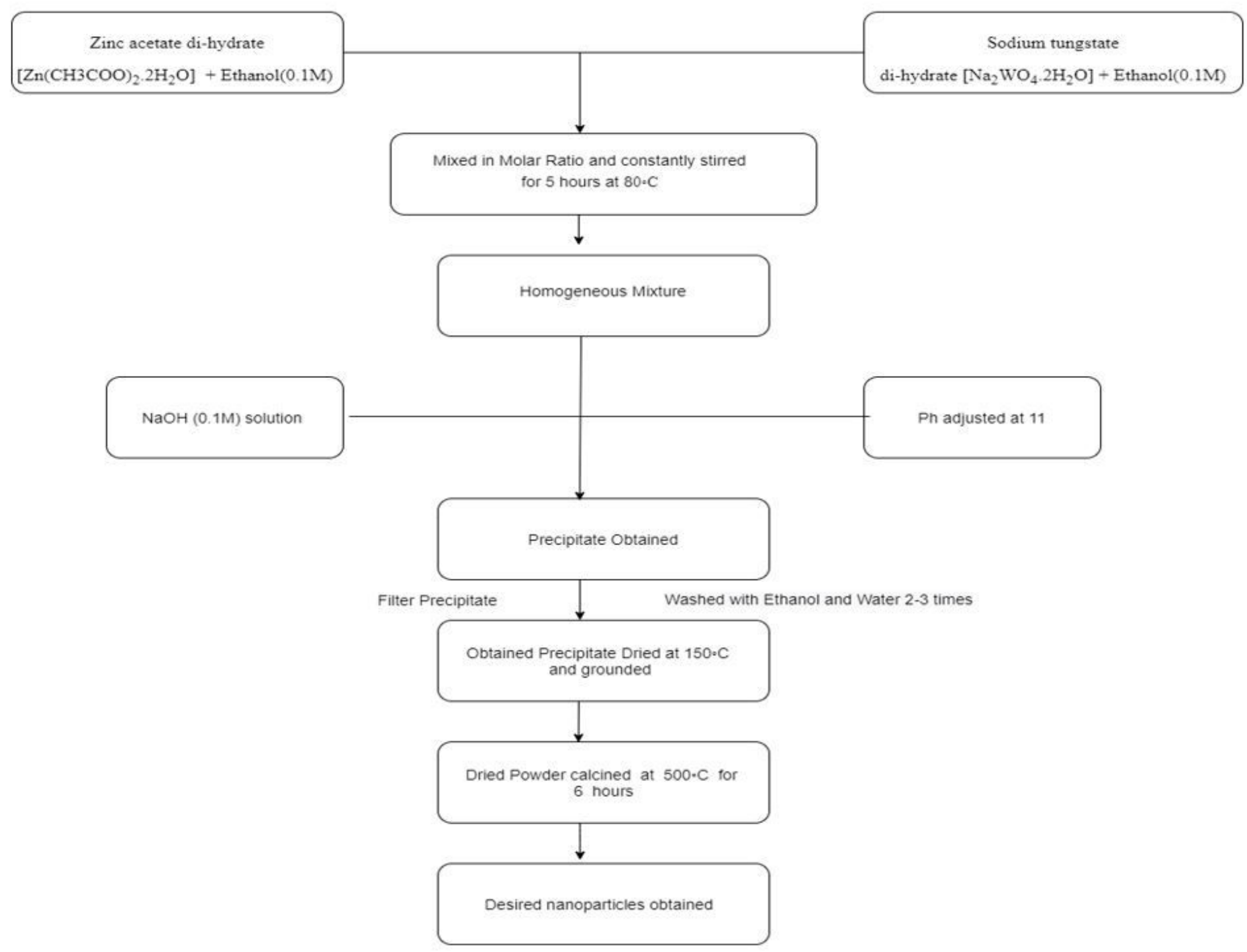

\section{Figure 1}

The schematic represntation co-precipitated $\mathrm{ZnO}$ nano particles. 

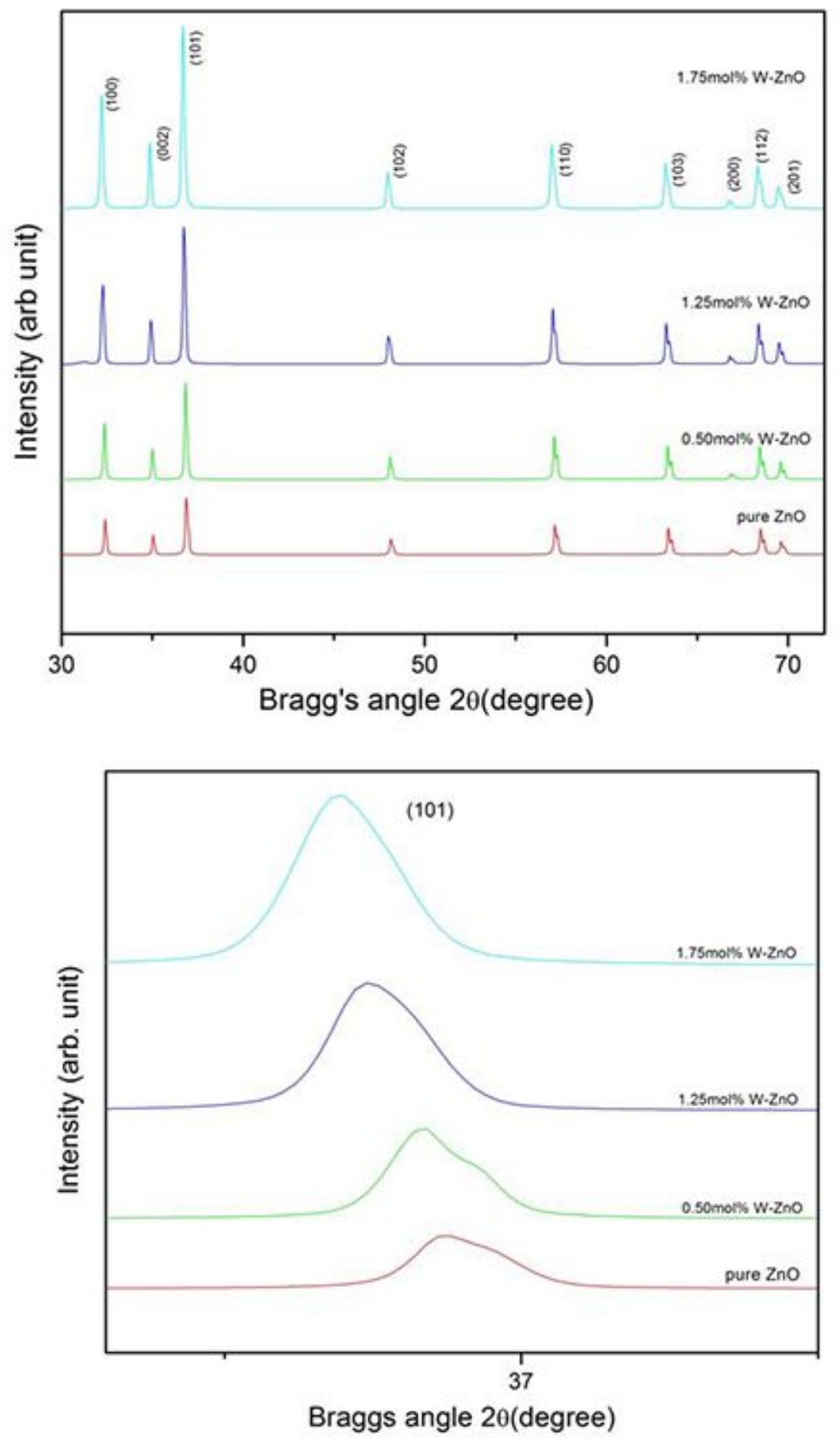

Figure 2

2a. XRD spectra of pure and W-ZnO. 2b XRD peak corresponding to plane (101) for W- ZnO samples. 


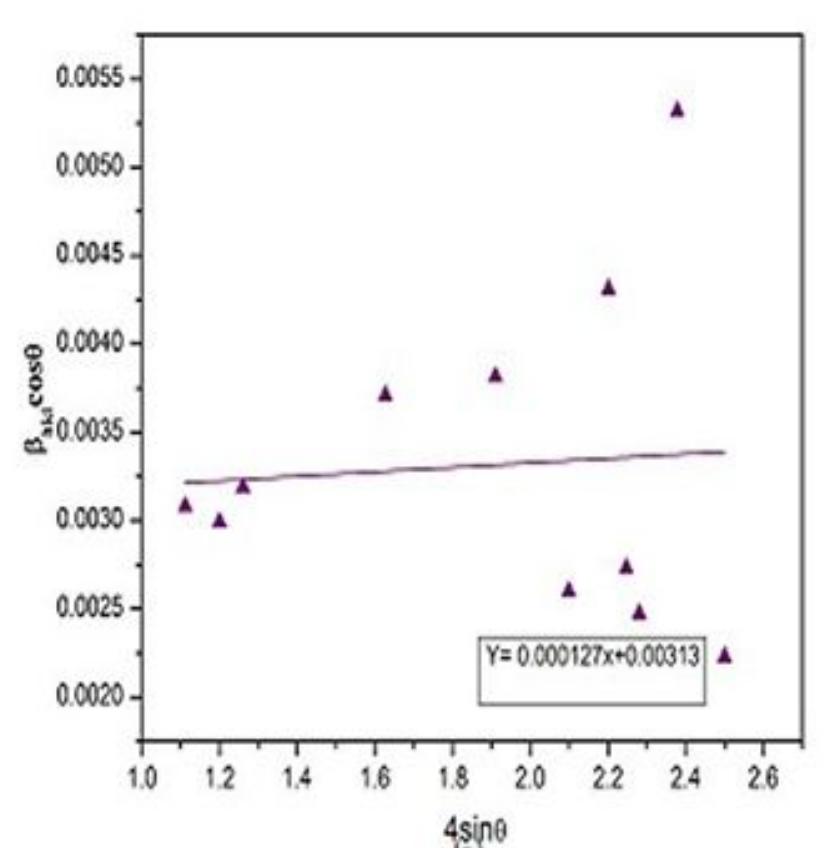

(a)

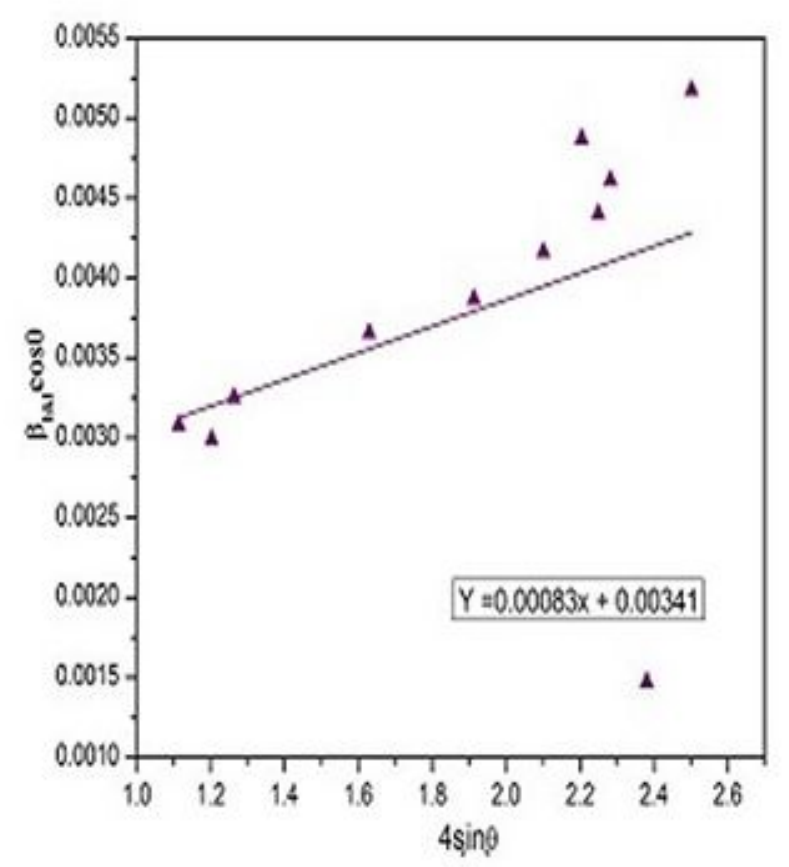

(c)

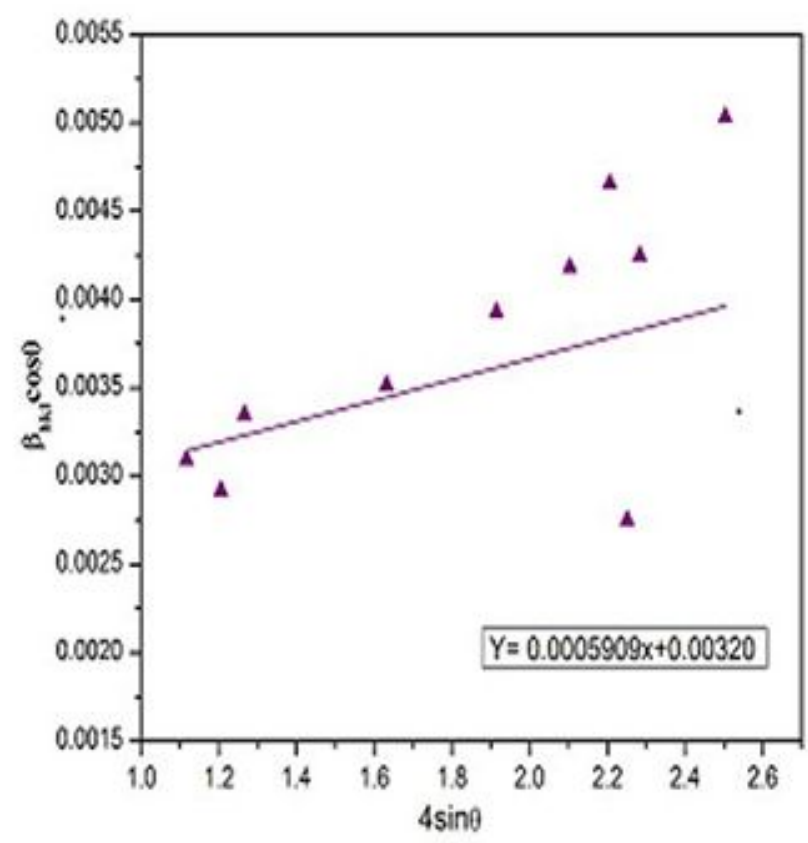

(b)

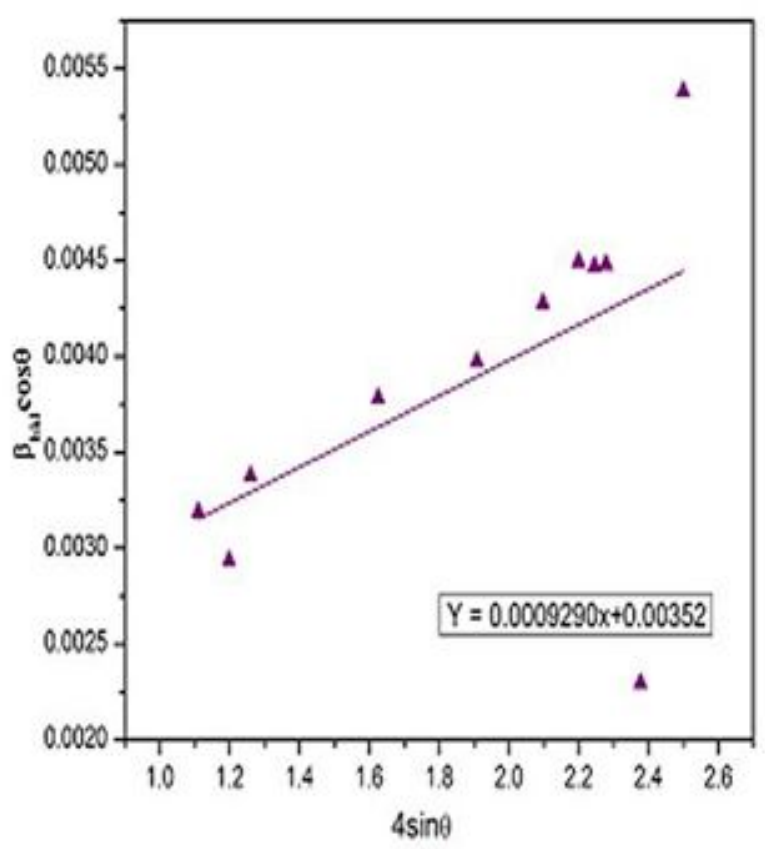

(d)

\section{Figure 3}

The W-H Analysis of undoped and doped ZnO Nano particles considering UDM (a) UDM for pure ZnO (b) UDM of $0.50 \mathrm{~mol} \% \mathrm{~W}-\mathrm{ZnO}$ (c) UDM of $1.25 \mathrm{~mol} \% \mathrm{~W}-\mathrm{ZnO}$ (d) UDM of $1.75 \mathrm{~mol} \% \mathrm{~W}-\mathrm{ZnO}$. 


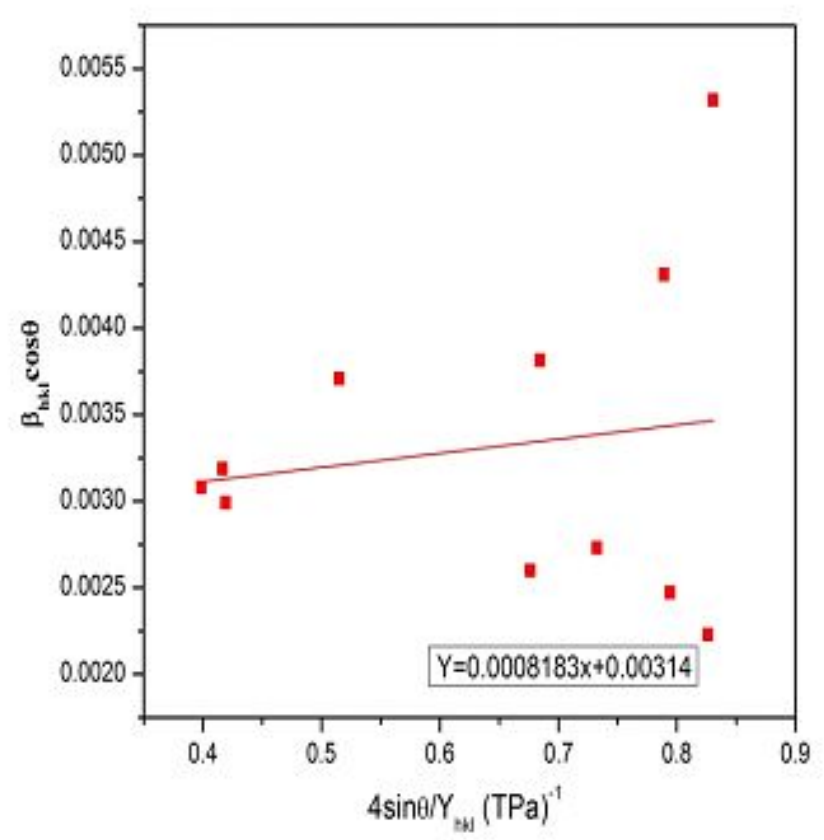

(a)

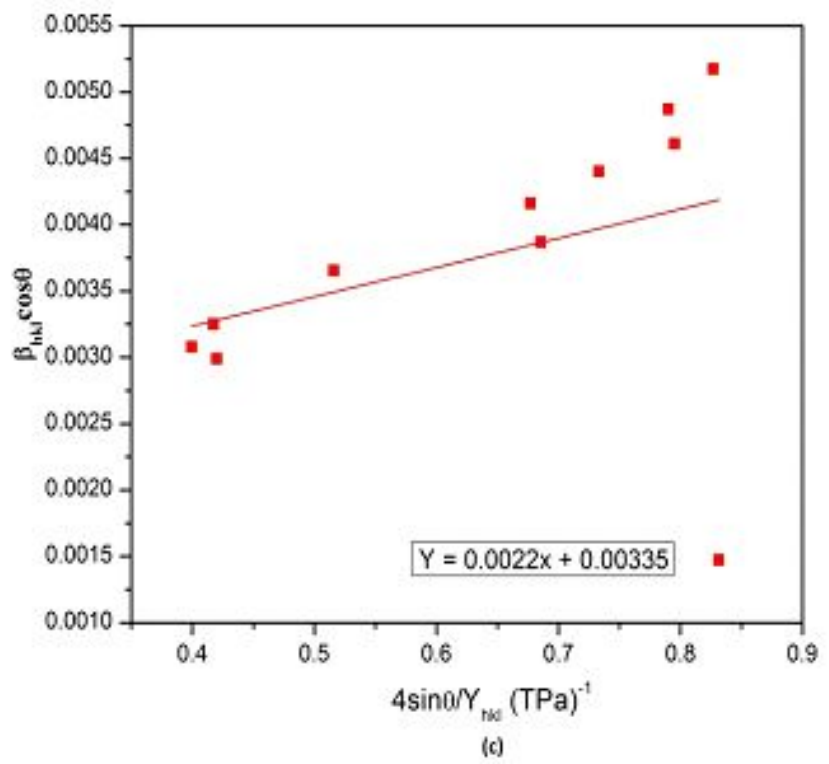

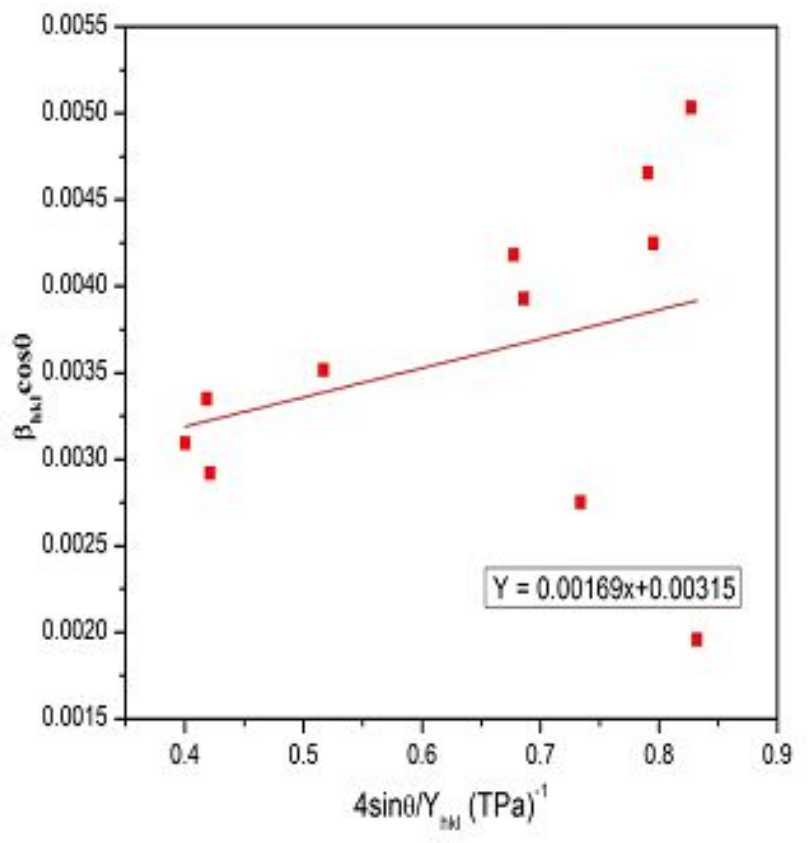

(b)

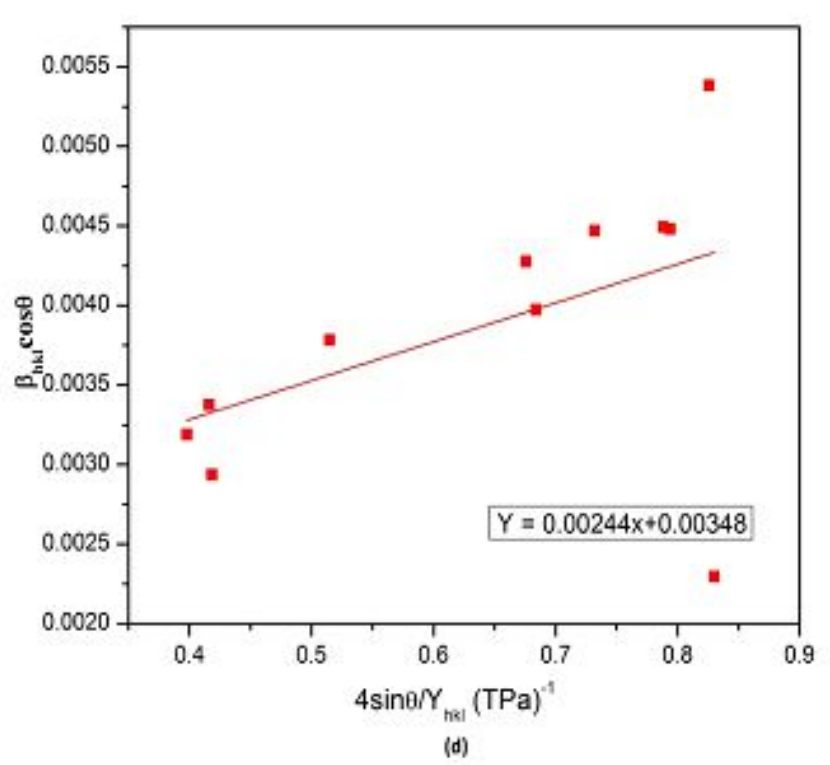

\section{Figure 4}

Remodeled W-H Analysis of undoped and doped ZnO Nano particles considering USDM. (a)USDM of pure $\mathrm{ZnO}$ (b) USDM of $0.50 \mathrm{~mol} \% \mathrm{~W}-\mathrm{ZnO}$ (c)USDM of $1.25 \mathrm{~mol} \% \mathrm{~W}-\mathrm{ZnO}$ (d)USDM of $1.75 \mathrm{~mol} \% \mathrm{~W}-\mathrm{ZnO}$. 

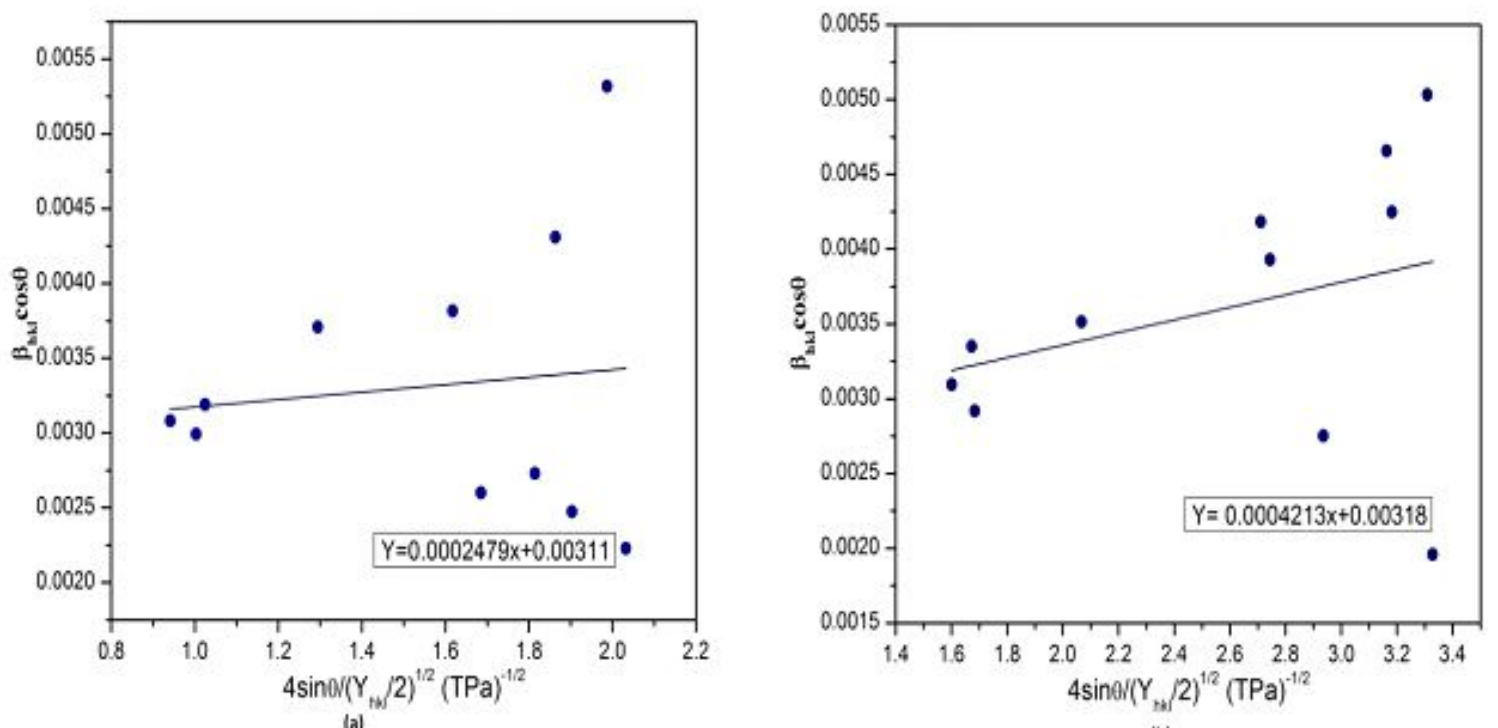

(b)

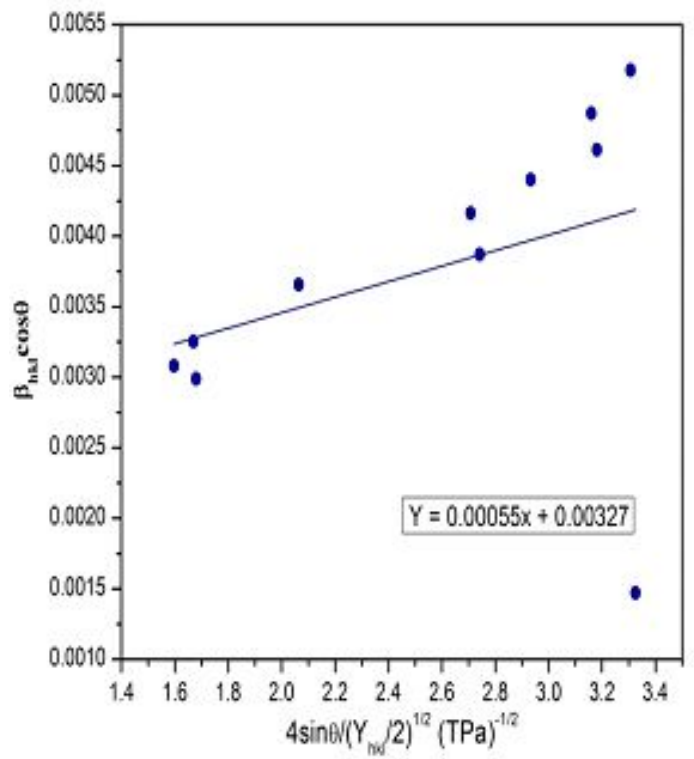

(c)

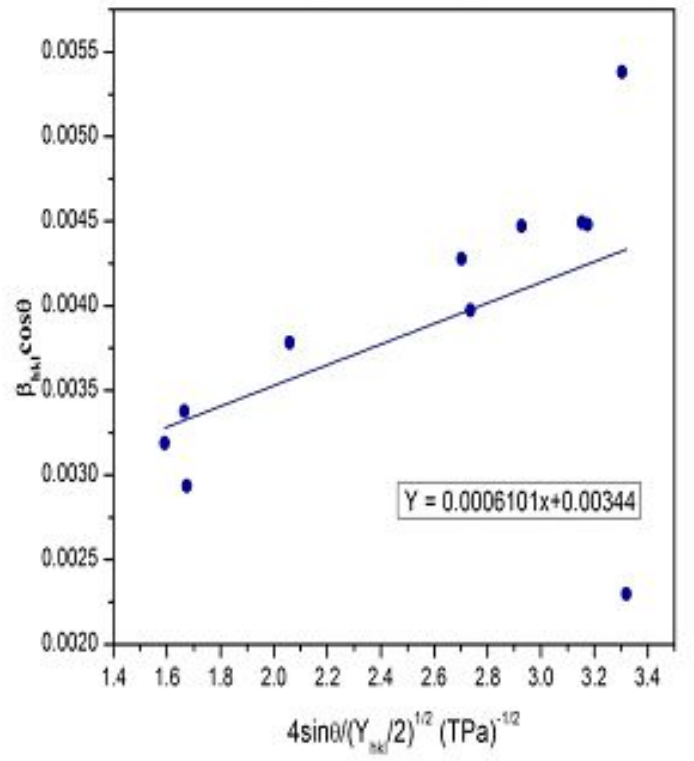

(d)

\section{Figure 5}

Remodeled W-H Analysis of undoped and doped ZnO Nano particles considering UDEDM (a)UDEDM of pure W-ZnO (b)UDEDM of $0.50 \mathrm{~mol} \% \mathrm{~W}-\mathrm{ZnO}$ (c)UDEDM of $1.25 \mathrm{~mol} \% \mathrm{~W}-\mathrm{ZnO}$ (d)UDEDM of $1.75 \mathrm{~mol} \% \mathrm{~W}$ $\mathrm{ZnO}$. 


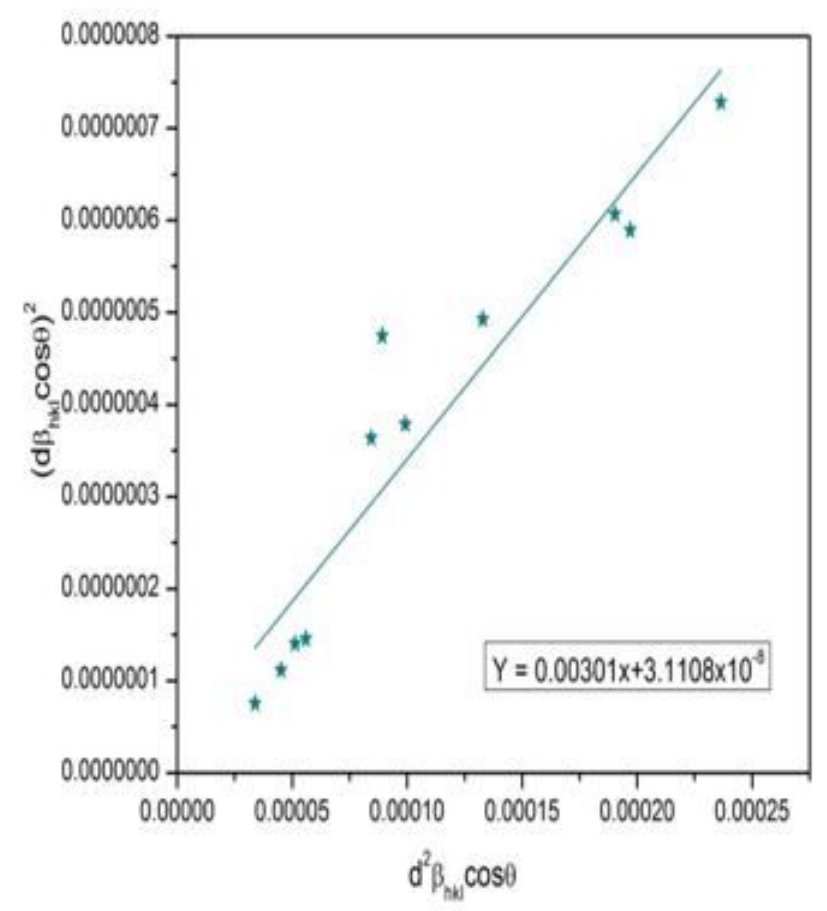

(a)

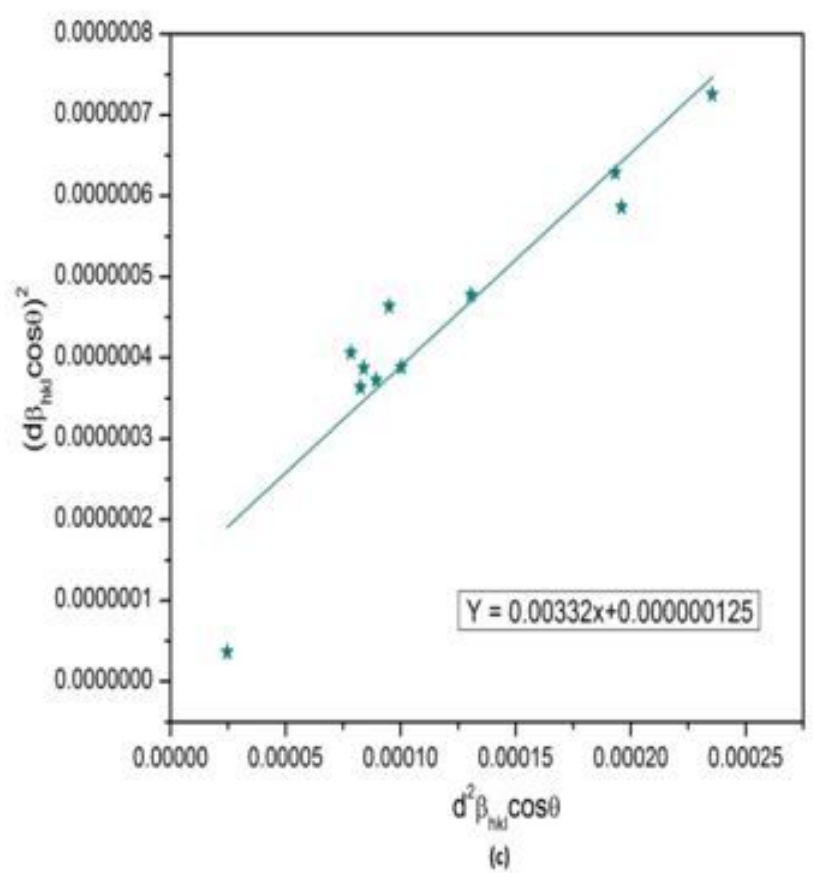

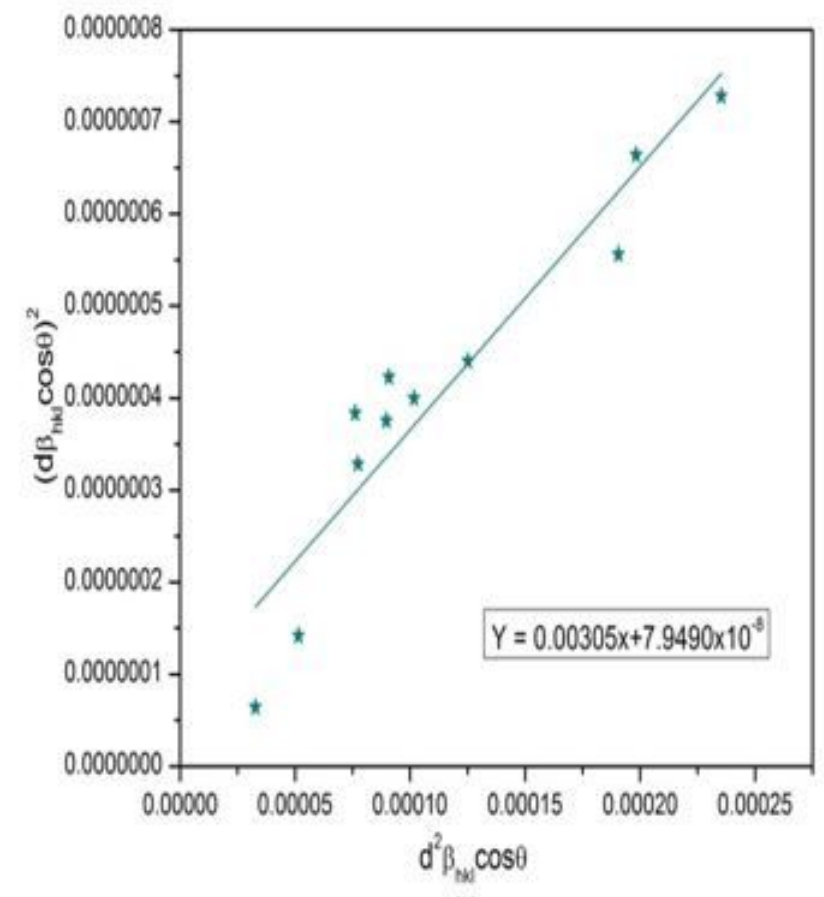

(b)

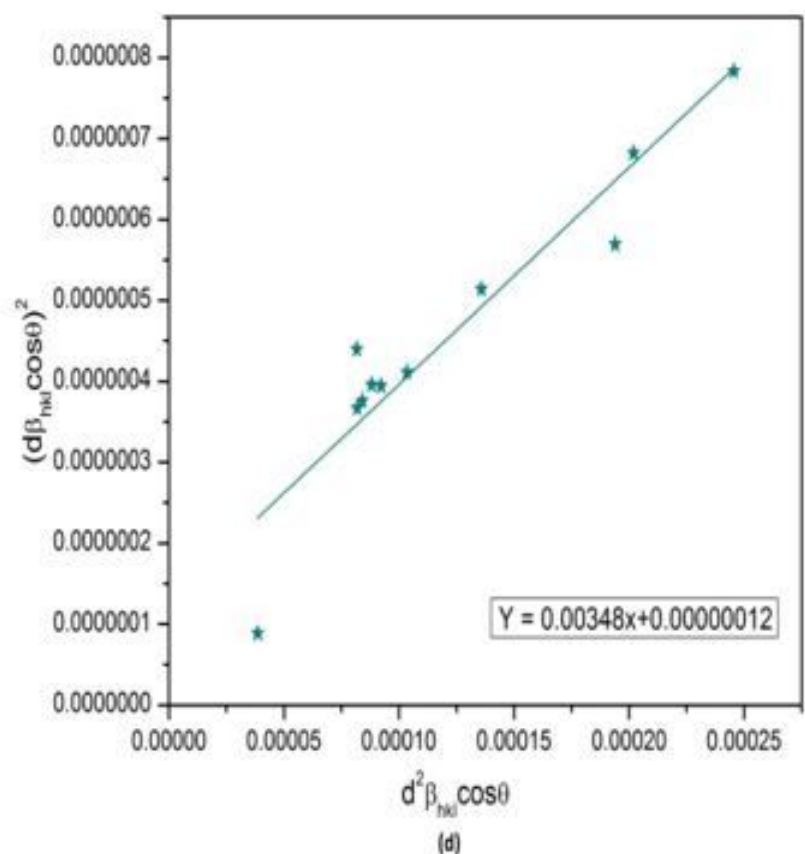

(d)

\section{Figure 6}

The SSP of undoped and doped ZnO Nano particles. (a) SSP of pure ZnO (b) SSP of $0.50 \mathrm{~mol} \% \mathrm{~W}-\mathrm{ZnO}$ (c) SSP of $1.25 \mathrm{~mol} \% \mathrm{~W}-\mathrm{ZnO}$ (d) SSP of $1.75 \mathrm{~mol} \% \mathrm{~W}-\mathrm{ZnO}$. 


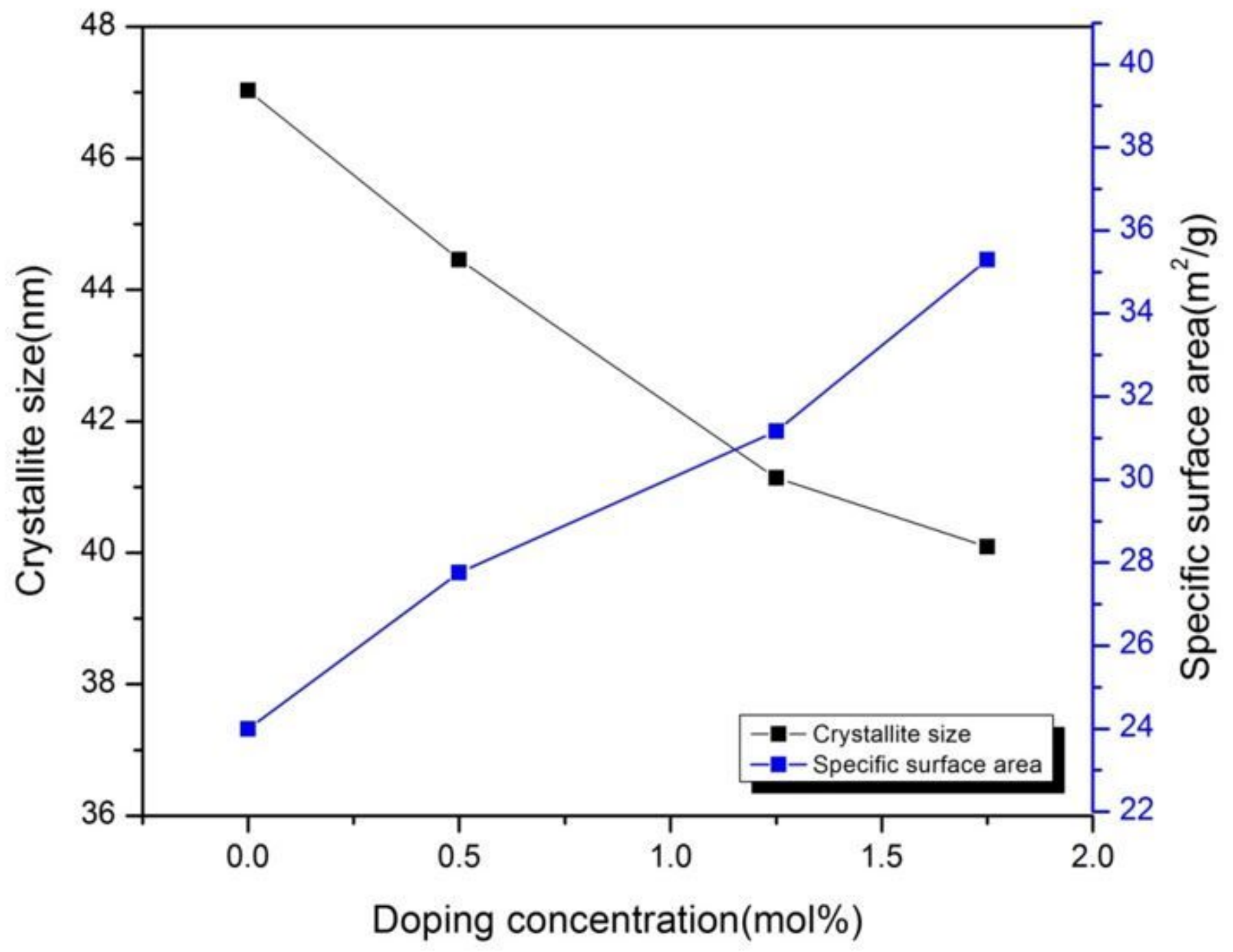

Figure 7

Curve depicting variation of crystallite size and specific surface area with doping concentration. 


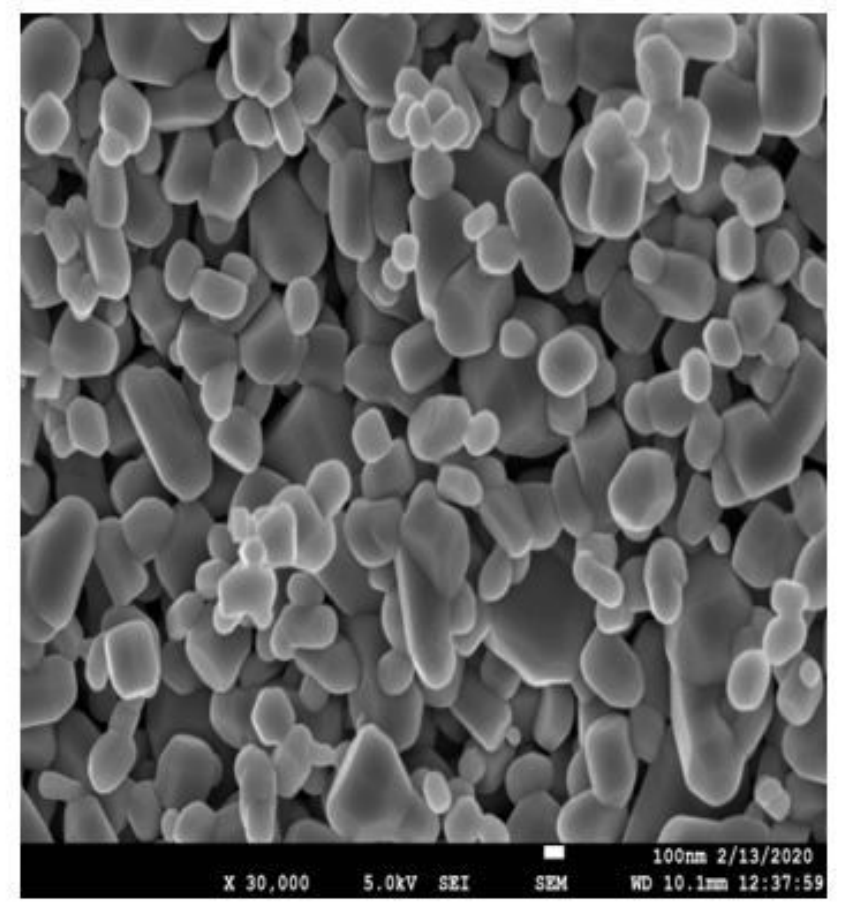

(a)

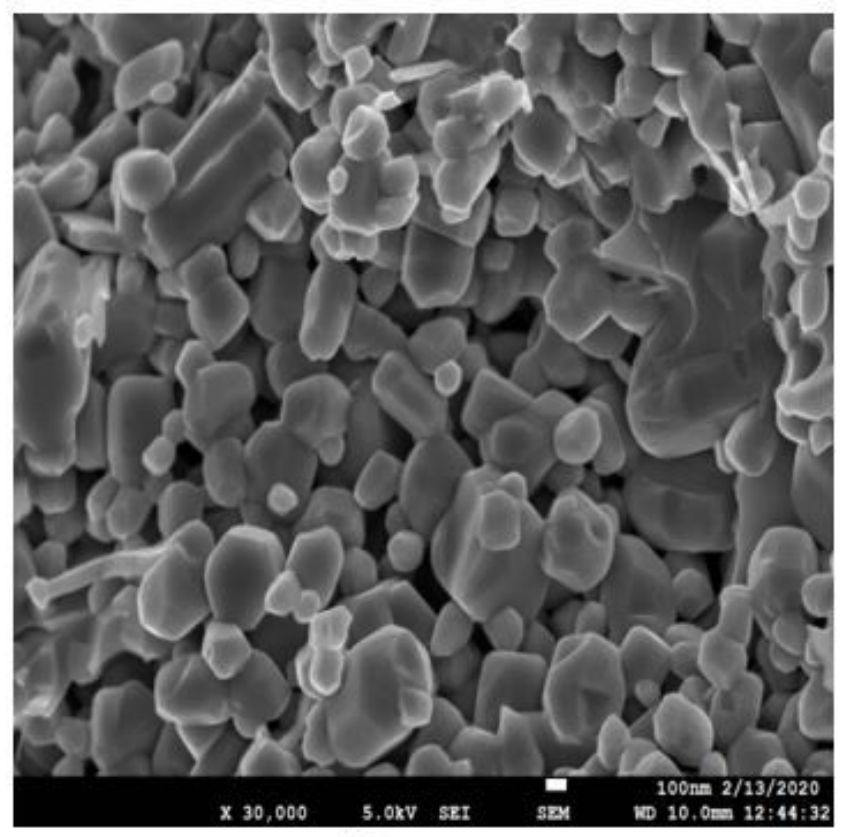

(c)

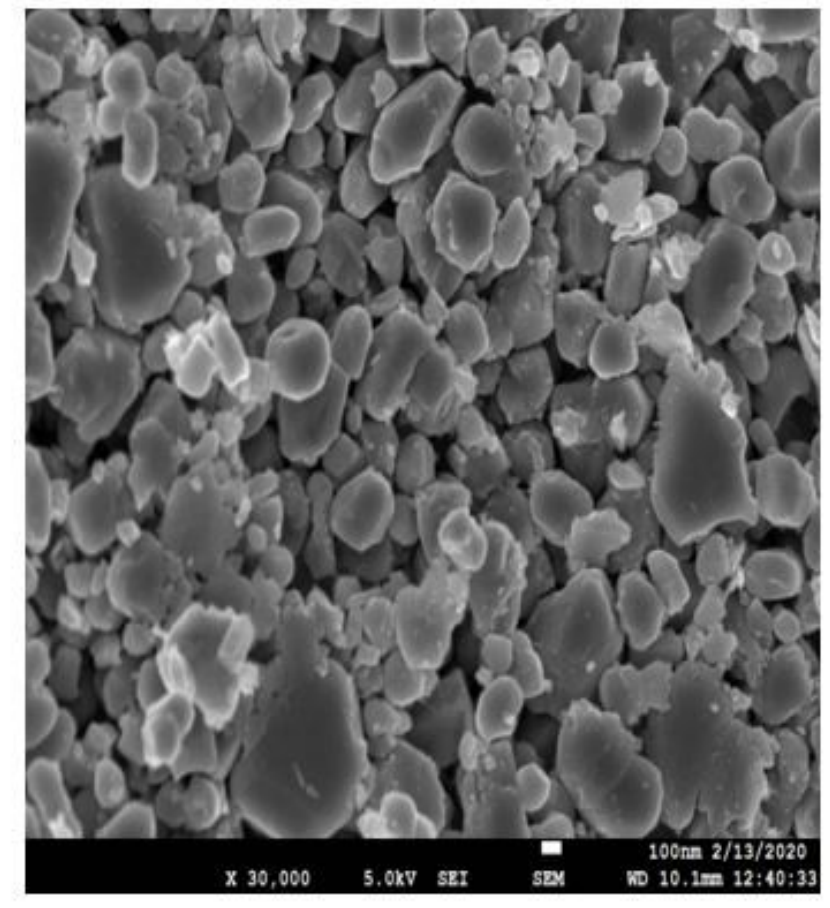

(b)

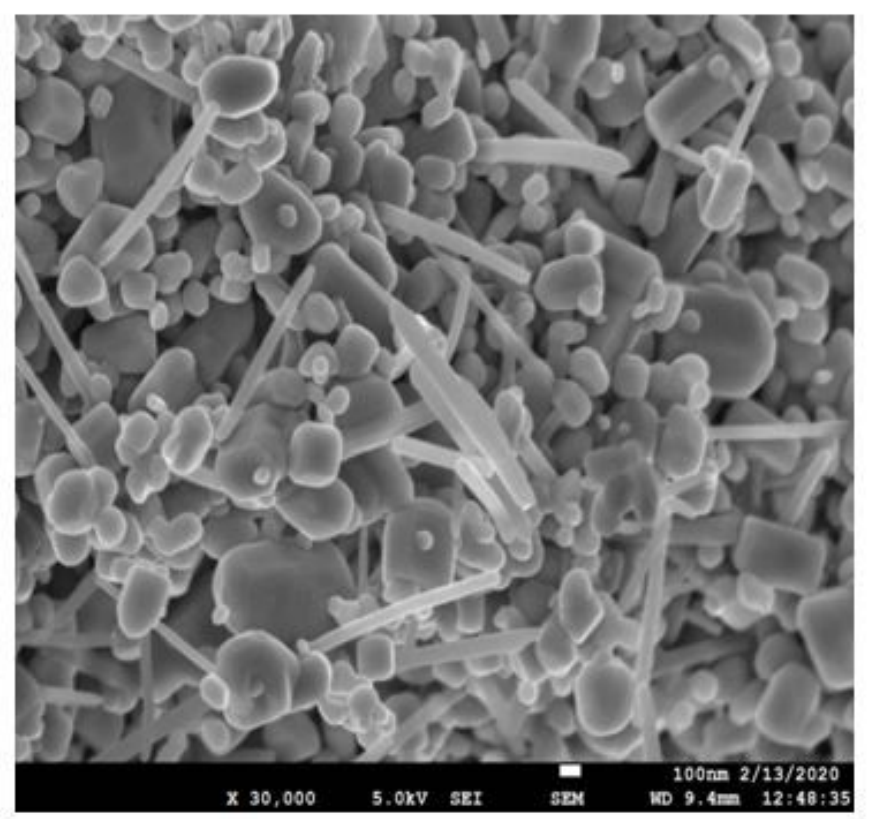

(d)

\section{Figure 8}

SEM micrographs of undoped and doped ZnO Nano particles. (a) Pure ZnO (b) $0.50 \mathrm{~mol} \% \mathrm{~W}-\mathrm{ZnO}$ (c) 1.25 mol\% W-ZnO (d) $1.75 \mathrm{~mol} \% \mathrm{~W}-\mathrm{ZnO}$ 


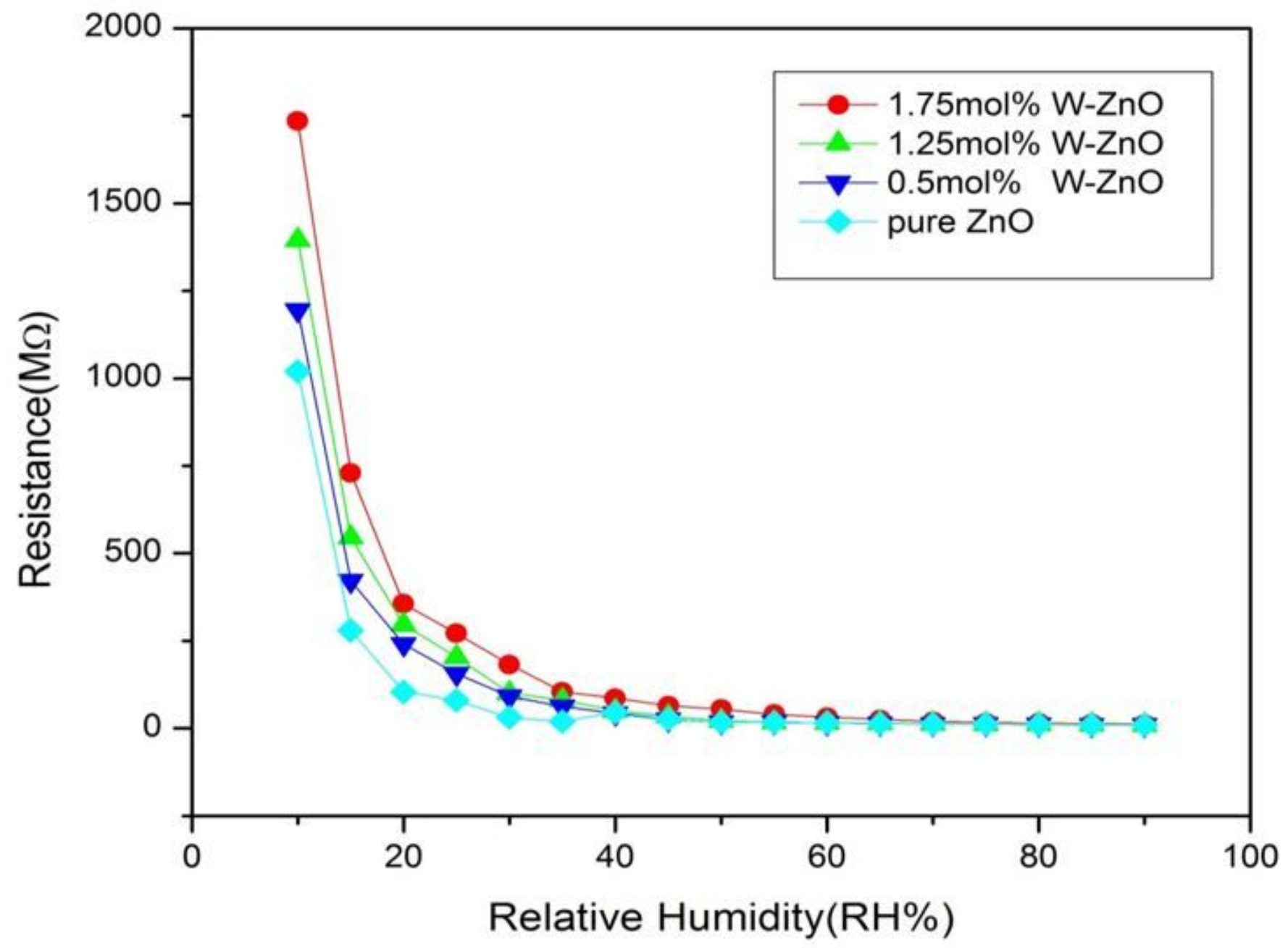

Figure 9

Relative humidity curve for pure and $\mathrm{W}$ doped $\mathrm{ZnO}$ nano-sensors. 


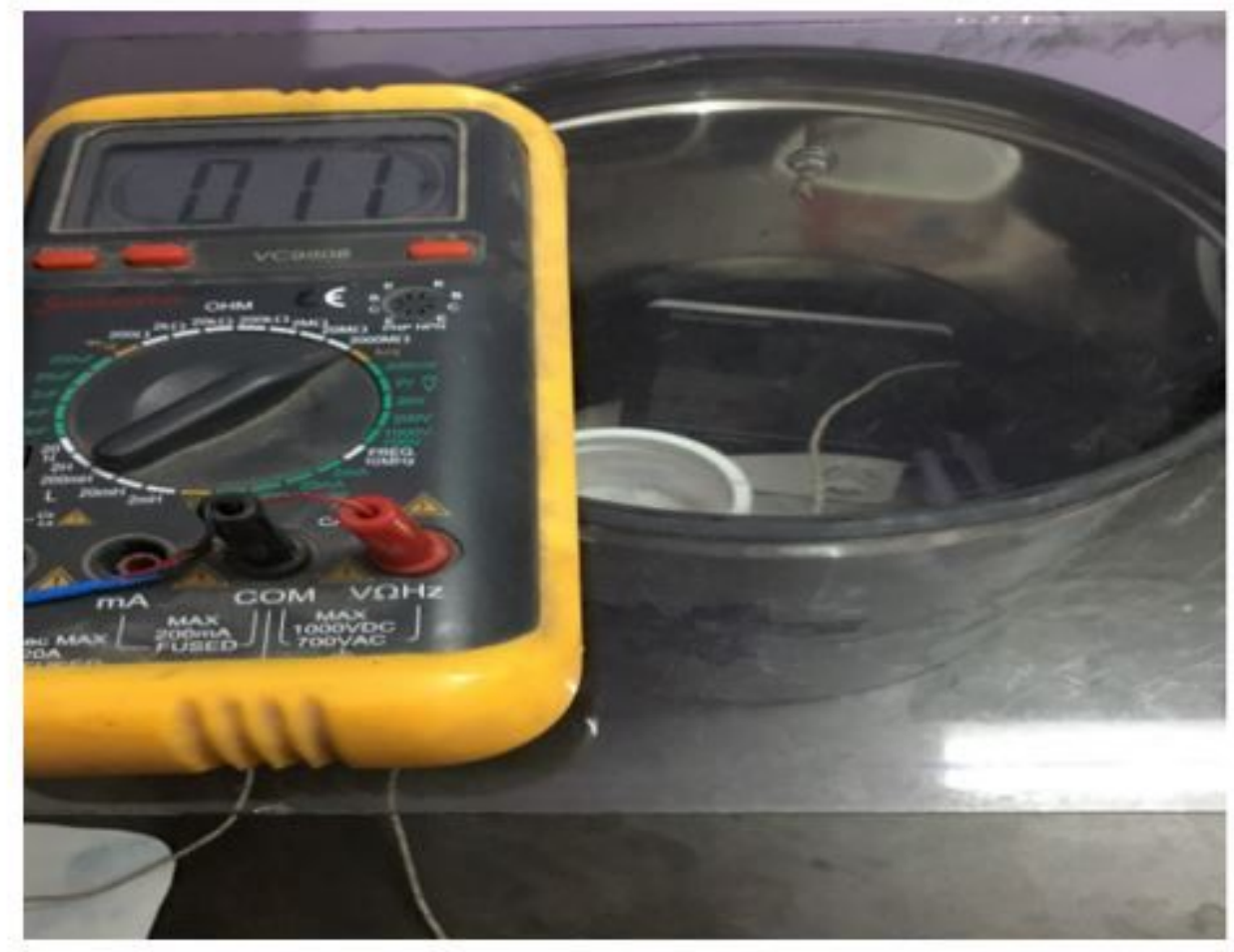

Figure 10

The experimental setup for Relative Humidity Sensing measurement. 


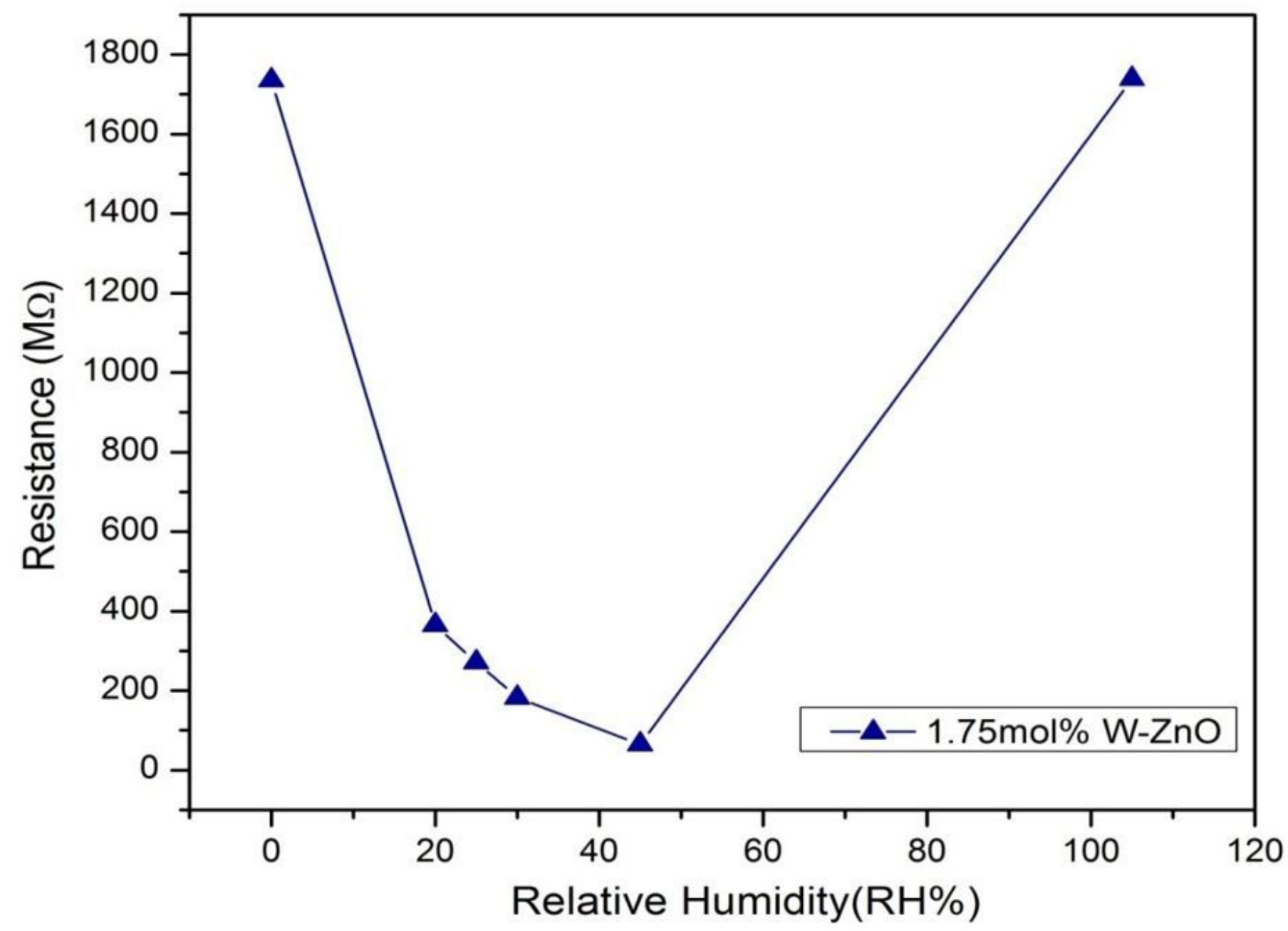

Figure 11

Response and Recovery time graph for $1.75 \mathrm{~mol} \% \mathrm{~W}-\mathrm{ZnO}$ humidity sensor. 


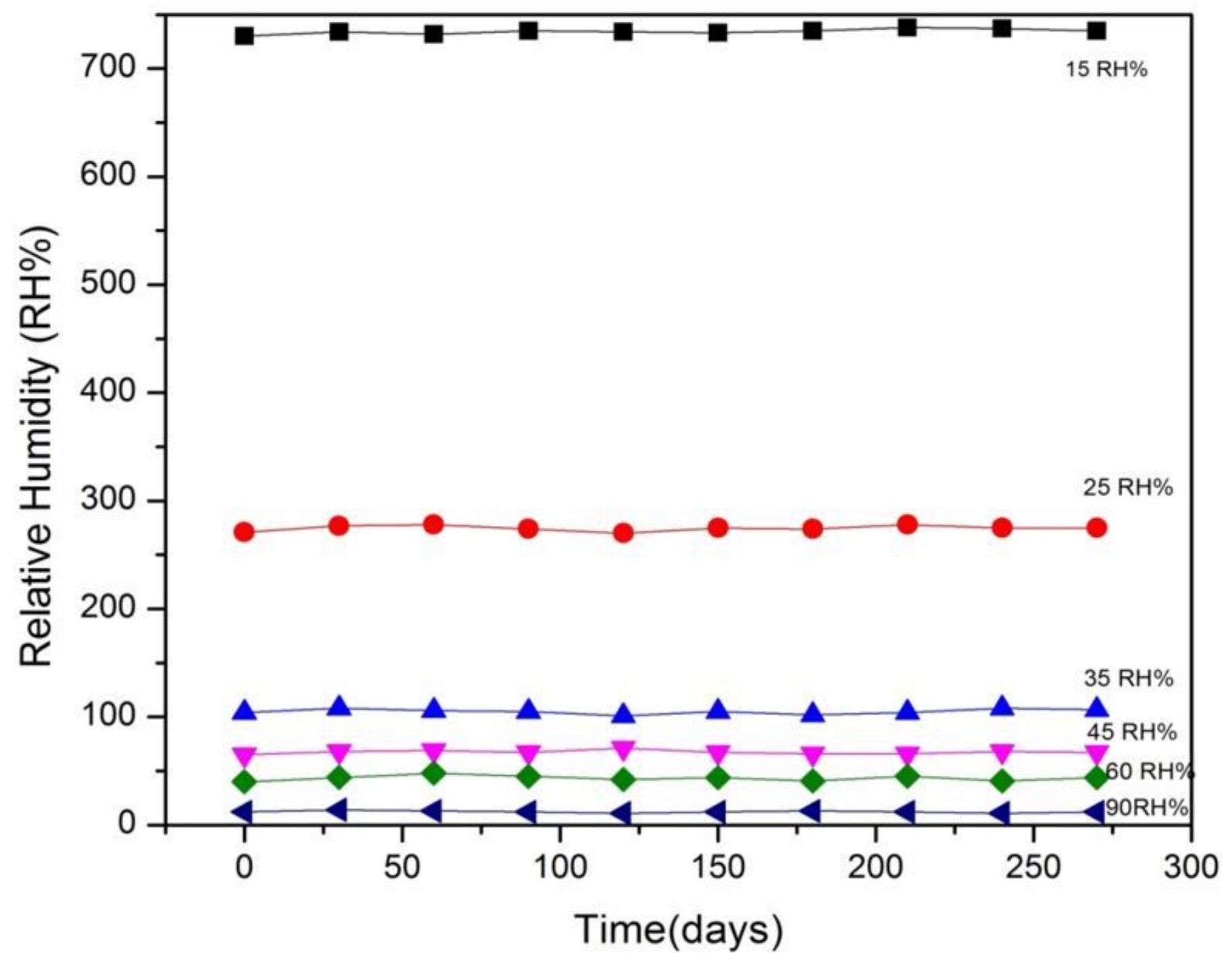

Figure 12

Stability Curve for $1.75 \mathrm{~mol} \% \mathrm{ZnO}$ sensor 


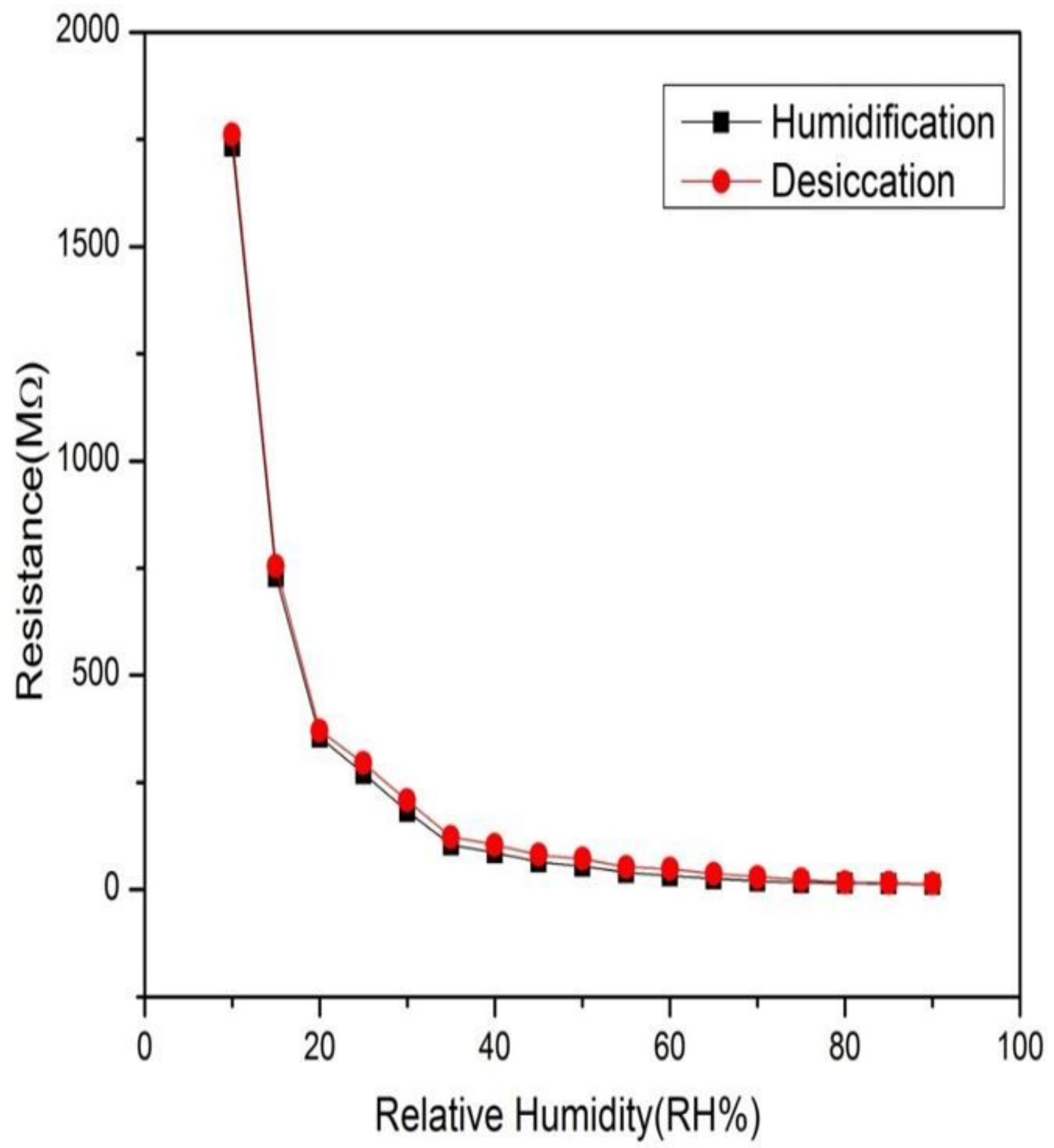

Figure 13

Hysteresis curve for $1.75 \mathrm{~mol} \% \mathrm{~W}$ doped $\mathrm{ZnO}$ nano-sensor. 